\title{
ON THE DEPTH OF THE SYMMETRIC ALGEBRA
}

\author{
J. HERZOG, ${ }^{1}$ M. E. ROSSI ${ }^{2}$ AND G. VALLA ${ }^{3}$
}

\begin{abstract}
Let $(R, \mathrm{~m})$ be a local ring. Assume that $R=A / I$, where $(A, \mathrm{n})$ is a regular local ring and $I \subseteq \mathrm{n}^{2}$ is an ideal. The depth of the symmetric algebra $S(\mathrm{~m})$ of $m$ over $R$ is computed in terms of the depth of the associated graded module $\operatorname{gr}_{11}(I)$ and the so-called "strong socle condition." Explicit results are obtained, for instance, if $I$ is generated by a super-regular sequence, if $I$ has a linear resolution or if $I$ has projective dimension one.
\end{abstract}

Introduction. In recent years the symmetric algebra $S(I)$ of an ideal $I$ and some related algebras, such as the Rees algebra $\mathscr{R}(I)$ of $I$, or the associated graded ring $\operatorname{gr}_{I}(R)$ of $I$, have been studied very extensively. One central problem always has been to give reasonable conditions for one of these algebras to be Cohen-Macaulay. As it turned out, the symmetric algebra has a strong tendency not to be CohenMacaulay. For instance, if we consider the symmetric algebra $S(\mathrm{~m})$ of the maximal ideal of a Cohen-Macaulay local ring $(R, \mathrm{~m})$ of positive dimension, Rossi [11] has shown that $S(\mathrm{~m})$ is Cohen-Macaulay if and only if $R$ is an abstract hypersurface ring; in other words, $S(\mathrm{~m})$ is almost never Cohen-Macaulay. One reason for this behavior of the symmetric algebra seems to be the fact that its dimension compared with the one of $R$ may be much higher. In their paper [7] Huneke and Rossi succeeded in giving a very explicit formula for the dimension $\operatorname{dim} S(M)$ of the symmetric algebra of a module $M$. Applied to the above situation one finds that $\operatorname{dim} S(\mathrm{~m})=$ embedding dimension of $R$, provided $R$ is not regular. (If $R$ is regular, then $\operatorname{dim} S(\mathrm{~m})=\operatorname{dim} R+1$.) It follows from a result in the same paper [7] that depth $S(m) \leqslant \operatorname{dim} R+1$. Here the gap between depth and dimension becomes apparent. Unfortunately the bound depth $S(\mathrm{~m}) \leqslant$ depth $R+1$ is not true in general if $R$ is not Cohen-Macaulay, see the remark after (2.12).

The main theme of the paper actually will be to compute or to estimate the depth of $S(\mathrm{~m})$ for a Noetherian local ring $(R, \mathrm{~m})$, in the range up to depth $R+1$. We shall see that depth $S(\mathfrak{m})$ is a much more sensitive invariant of $S(\mathfrak{m})$ than its dimension.

Before going into a detailed description of our paper we just quote two of our results to give an impression of how delicate depth $S(\mathfrak{m})$ behaves.

Received by the editors July 29, 1985.

1980 Mathematics Subject Classification. Primary 13D25; Secondary $13 \mathrm{H} 10$.

${ }^{1}$ Partially supported by the GMD-CNP ${ }_{q}$ Exchange Program.

${ }^{2}$ Member of G.N.S.A.G.A. of C.N.R., Italy.

${ }^{3}$ Partially supported by $\mathrm{CNP}_{\mathrm{q}}$.

(C1986 American Mathematical Society $0002-9947 / 86 \$ 1.00+\$ .25$ per page 
In (3.7) we give an example of a two-dimensional complete intersection domain $(R, \mathfrak{m})$ for which depth $S(\mathfrak{m})=0$. On the other hand, we show in (5.1) that if $(R, \mathrm{~m})$ is a quotient of a regular local ring by a super-regular sequence, then

$$
\text { depth } R \leqslant \operatorname{depth} S(\mathrm{~m}) \leqslant \operatorname{depth} R+1 \text {. }
$$

Even though, in both cases, $R$ is a complete intersection, depth $S(\mathrm{~m})$ behaves quite differently. The reason for this feature is that depth $S(\mathrm{~m})$ reflects more the algebraic nature of $\operatorname{gr}_{\mathrm{m}}(R)$ than the one of $R$ itself.

Throughout the paper we deal with the following situation: $(A, \mathfrak{n})$ is a regular local ring with an infinite residue class field $k$, and $R=A / I$ is the local ring with maximal ideal $m$ and depth $d$, where $I \subseteq \mathfrak{n}^{2}$ is an ideal in $A$. The crucial idea of the paper is the observation that $\operatorname{gr}_{\mathfrak{n}}(I)=\oplus_{p \geqslant 0} I \mathfrak{n}^{p} / I \mathfrak{n}^{p+1}$ sits inside $S(\mathrm{~m})$ as a graded submodule. In fact, we notice in (2.2) that $S(\mathrm{~m})=\oplus_{p \geqslant 0} \mathrm{n}^{p} / I \mathrm{n}^{p-1}$, so $I \mathfrak{n}^{p} / I \mathfrak{n}^{p+1} \subseteq \mathfrak{n}^{p+2} / I \mathfrak{n}^{p+1}$ for all $p \geqslant 0$. Thus we get a canonical embedding $\operatorname{gr}_{11}(I)(-2) \rightarrow S(n)$. This embedding relates the depth of $S(\mathrm{~m})$ with the depth of $\operatorname{gr}_{11}(I)$. The advantage of this comparison is that depth $\operatorname{gr}_{\mathfrak{n}}(I)$ is much more accessible than depth $S(\mathrm{~m})$.

In $\S 1$ we collect some generalities on filtrations, graded rings, and modules that will be used in the paper.

The main theorem (2.13) in the paper-the comparison of depth $\operatorname{gr}_{n}(I)$ and depth $S(\mathrm{~m})$-is proved in $\S 2$. The result is this: (a) If $\operatorname{depth} \operatorname{gr}_{\mathrm{n}}(I) \leqslant d$, then depth $S(\mathfrak{m})=$ depth $\operatorname{gr}_{\mathfrak{n}}(I)$. (b) If depth $\operatorname{gr}_{\mathfrak{n}}(I)=d+1$, then depth $S(\mathfrak{m}) \geqslant d$.

The third part of the theorem deals with the question, when is depth $S(\mathrm{~m}) \geqslant d+$ 1? To decide this, depth $\operatorname{gr}_{n}(I)$ is not the only measure. The other ingredient that determines whether depth $S(\mathrm{~m}) \geqslant d+1$ is given by the so-called "strong socle condition." The ring $R=A / I$ is said to have a $t$-strong socle if $I n^{t}: n \subseteq I . R$ is said to have strong socle if it has a 1-strong socle.

Suppose $R$ has a strong socle, and let $x \in A$ be an element that corresponds to a nonzero element of the socle of $R$. Then $x \mathfrak{n} \subseteq I$, but $x \notin I$. This explains the terminology "strong socle."

In the third part of our main theorem we roughly show this: (c) depth $S(\mathrm{~m}) \geqslant d$ +1 if and only if depth $\operatorname{gr}_{\mathrm{n}}(I) \geqslant d+1$ and there exists a "nice" regular sequence $x_{1}, \ldots, x_{d}$ in $R$ such that $R /\left(x_{1}, \ldots, x_{d}\right)$ has a $d$-strong socle. The attribute "nice" has an explicit technical meaning which we do not want to explain now. The definition is given in $\$ 2$.

The use of the theorem obviously depends on the ability to compute depth $\operatorname{gr}_{n}(I)$ and to check the strong socle condition. The depth of $\operatorname{gr}_{\mathfrak{n}}(I)$ is studied in $\S 3$. If $M$ is a finitely generated module over the regular local ring $(A, \mathfrak{n})$ with a stable $\mathfrak{n}$-filtration $\mathscr{F}$, then $\operatorname{gr}_{\mathscr{F}}(M)$ is a graded $P$-module, where $P=\operatorname{gr}_{\mathfrak{n}}(A)$. For the Betti numbers of $M$ and $\operatorname{gr}_{\mathscr{F}}(M)$ one easily proves $\beta_{i}\left(\operatorname{gr}_{\mathscr{F}}(M)\right) \geqslant \beta_{i}(M)$ for every $i \geqslant 0$. It follows that depth $\operatorname{gr}_{\mathscr{F}}(M) \leqslant \operatorname{depth} M$. In particular, if $I$ is an ideal, then

$$
\text { depth } \operatorname{gr}_{\mathrm{n}}(I) \leqslant d+1 .
$$


We call $M$ of homogeneous type if $\beta_{i}(M)=\beta_{i}\left(g_{\mathfrak{n}}(M)\right)$ for every $i \geqslant 0$. In connection with our main theorem (2.3) it is interesting to know when $I$ is of homogeneous type, since then depth $\operatorname{gr}_{\mathfrak{n}}(I)=d+1$, and depth $S(\mathrm{~m}) \geqslant d$.

There are two interesting classes of ideals which are of homogeneous type: (1) ideals which have a linear resolution; (2) ideals generated by super-regular sequences, see (3.6). To prove (2) we use a splitting theorem (3.5) for $\operatorname{gr}_{\mathfrak{n}}(I)$ : If $I=J+K$ for some ideals $J$ and $K$, and if $I \cap \mathfrak{n}^{t}=J \mathfrak{n}^{t-v(K)+1}$ for all $t \geqslant 0$, then $\operatorname{gr}_{n}(I)=\operatorname{gr}_{n}(J) \oplus \operatorname{gr}_{n}(I / J)$. This theorem provides in many cases an inductive procedure for calculating $\operatorname{gr}_{\mathfrak{n}}(I)$. We conclude $\$ 3$ by explicitly discussing $\operatorname{gr}_{\mathfrak{n}}(I)$ when the homological dimension of $I$ is 1 .

$\S 4$ is devoted to studying the strong socle condition. Here we assume that $(A, \mathfrak{n})$ is a regular local ring, and $R=A / I$ is of depth 0 , where $I \subseteq \mathfrak{n}^{2}$. It is clear from the definition that $R$ has a $t$-strong socle for $t \gg 0$. A lower bound for such a $t$ can be given quite generally in terms of invariants of $R$ and $I$. A very precise statement can be made for an ideal $I$ generated by a super-regular sequence $f_{1}, \ldots, f_{n}$. Let $v_{i}$ be the value of $f_{i}$ with respect to the $n$-adic filtration. We may assume that $v_{1} \geqslant v_{2} \geqslant$ $\cdots \geqslant v_{n}$. In (4.7) we show that $R=A / I$ has a $t$-strong socle if and only if $t \geqslant \sum_{i=2}^{n} v_{i}-n+2$. It follows from this estimate that such a ring can have a strong socle only if $I$ is principal. Surprisingly we even can show that a Gorenstein ring has a strong socle if and only if it is a hypersurface ring; see (4.13). Quite to the contrary $R$ has always a strong socle if $I$ has a linear resolution, as we show in (4.14). Again, if the homological dimension of $I$ is 1 , the picture becomes clear. Here we can show the following conditions to be equivalent (4.18): (1) $R$ has a strong socle and is of homogeneous type. (2) $I$ has a linear resolution.

In the last section (§5) we apply the results proved before to compute depth $S(\mathrm{~m})$ for some classes of local rings. As before we get a complete answer if $I$ is generated by a super-regular sequence or if $I$ has a linear resolution. In the first case $d \leqslant \operatorname{depth} S(\mathrm{~m}) \leqslant d+1$; in the second case depth $S(\mathrm{~m}) \geqslant d+1$. Moreover, in the first case depth $S(\mathrm{~m})=d+1$ if and only if $d \geqslant \sum_{i=2}^{n} v_{i}-n+2$, with the notations as above. These results can be found in (5.1) and (5.2).

Among many other applications we finally point out the discussion of depth $S(\mathrm{~m})$ for a monomial space curve $R=k\left[\left|t^{a}, t^{b}, t^{c}\right|\right] \simeq k[|X, Y, Z|] / I$. Here the defining ideal $I$ is of homological dimension 1 . We can show that $1 \leqslant \operatorname{depth} S(m) \leqslant 2$, and we are able to give necessary and sufficient numerical conditions for the equality $\operatorname{depth} S(\mathfrak{m})=2$.

The authors want to thank A. Simis and W. V. Vasconcelos for many stimulating discussions in connection with this paper.

\section{Preliminaries on filtered modules.}

(1.1) Let $(A, \mathfrak{n})$ be a Noetherian local ring and $M$ a finitely generated $A$-module. We say, accordingly to [1], that a filtration $\mathscr{F}=\left\{\mathscr{F}_{p} M\right\}_{p \geqslant 0}$ on $M$ is an n-filtration if $\mathfrak{n} \mathscr{F}_{p} M \subseteq \mathscr{F}_{p+1} M$ for all $p \geqslant 0$, and a stable $\mathfrak{n}$-filtration if $\mathfrak{n} \mathscr{F}_{p} M=\mathscr{F}_{p+1} M$ for all sufficiently large $p$. 
In the following a filtered $A$-module will be always an $A$-module together with a stable $\mathfrak{n}$-filtration $\mathscr{F}$.

A typical example of a stable $n$-filtration is the $n$-adic filtration which is defined as follows: $\mathscr{F}_{p} M=\mathfrak{n}^{p} M, p \geqslant 0$.

Given a filtered $A$-module $M$ we may consider the module $\operatorname{gr}_{\mathscr{F}}(M)=$ $\oplus_{p \geqslant 0}\left(\mathscr{F}_{p} M / \mathscr{F}_{p+1} M\right)$, which has a natural structure of a $\operatorname{gr}_{\mathfrak{n}}(A)$-module, where we use the convention that, if $\mathscr{F}$ is the $n$-adic filtration, we simply write $\operatorname{gr}_{\mathrm{n}}(M)$ instead of $\operatorname{gr}_{\mathscr{F}}(M)$.

(1.2) If $M$ is a filtered $A$-module and $m \in M$, we denote by $v_{\mathscr{F}}(m)$ the largest integer $p$ such that $m \in \mathscr{F}_{p} M$. We have $v_{\mathscr{F}}(m)<\infty$, and we denote by $m^{*}$ the residue class of $m$ in $\mathscr{F}_{p} M / \mathscr{F}_{p+1} M$ and call it the $\mathscr{F}$-initial form of $m$. Further, if $N$ is a submodule of a filtered $A$-module $M$, we get induced natural filtrations on $N$ and $M / N$ which are again stable n-filtrations: $\mathscr{F}_{p} N=N \cap \mathscr{F}_{p} M$ and $\mathscr{F}_{p}(M / N)=$ $\left(\mathscr{F}_{p} M+N\right) / N$.

The graded module of $N$ with respect to the induced filtration will be denoted simply by $N^{*}$. In fact, it is clear that $N^{*}$ is the submodule of $\operatorname{gr}_{\mathscr{F}}(M)$ generated over $\operatorname{gr}_{n}(A)$ by $x^{*}, x$ running in $N$.

(1.3) If $M$ and $N$ are filtered $A$-modules and $f: M \rightarrow N$ an $A$-homomorphism, $f$ is said to be a morphism of filtered modules if $f\left(\mathscr{F}_{p} M\right) \subseteq \mathscr{F}_{p} N$.

The morphism of filtered modules $f: M \rightarrow N$ clearly induces a morphism of graded $\operatorname{gr}_{n 1}(A)$-modules $\operatorname{gr}(f): \operatorname{gr}_{\mathscr{F}}(M) \rightarrow \operatorname{gr}_{\mathscr{F}}(N)$. It is clear that $\operatorname{gr}_{\mathscr{F}}(\cdot)$ is a functor from the category of filtered $A$-modules into the category of graded $\operatorname{gr}_{n}(A)$-modules.

Furthermore, we have a canonical embedding $(\operatorname{Ker} f)^{*} \rightarrow \operatorname{Ker} \operatorname{gr}(f)$.

(1.4) Let $f: M \rightarrow N$ be a morphism of filtered modules; $f$ is said to be strict if $f\left(\mathscr{F}_{p} M\right)=f(M) \cap \mathscr{F}_{p} N$.

The relevance of this notion is in the following result. Let $\mathbf{F}: M \stackrel{g}{\rightarrow} N \stackrel{f}{\rightarrow} P$ be a complex of filtered modules and

$$
\operatorname{gr}(\mathbf{F}): \operatorname{gr}_{\mathscr{F}}(M) \stackrel{\operatorname{gr}(g)}{\rightarrow} \operatorname{gr}_{\mathscr{F}}(N) \stackrel{\operatorname{gr}(f)}{\rightarrow} \operatorname{gr}_{\mathscr{F}}(P)
$$

be the induced complex of graded $\operatorname{gr}_{\mathfrak{n}}(A)$-modules. Then $\operatorname{gr}(\mathbf{F})$ is exact if and only if $\mathbf{F}$ is exact and $f$ and $g$ are strict morphisms.

Thus if $N$ is a submodule of $M$, using the fact that the embedding $i: N \rightarrow M$ and the projection $p: M \rightarrow M / N$ are strict morphisms with respect to the induced filtrations, we get that $\operatorname{gr}_{\mathscr{F}}(M / N)=\operatorname{gr}_{\mathscr{F}}(M) / N^{*}$.

As a further consequence of the above result, we get that a morphism $f: M \rightarrow N$ of filtered modules is strict if and only if the canonical embedding $(\operatorname{Ker} f)^{*} \rightarrow$ $\operatorname{Ker} \operatorname{gr}(f)$ is an isomorphism.

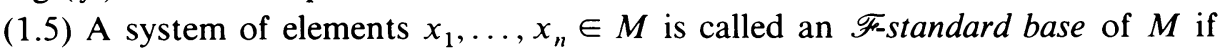
$\operatorname{gr}_{\mathscr{F}}(M)$ is generated over $\operatorname{gr}_{\mathrm{n}}(A)$ by $x_{1}^{*}, \ldots, x_{n}^{*}$. It is easy to prove that any standard base of $M$ is a system of generators of $M$.

Let $\varphi: A^{s} \rightarrow M$ be a free presentation of the filtered $A$-module $M$, and let $e_{1}, \ldots, e_{s}$ be the natural basis of $A^{s}$. If $x_{i}=\varphi\left(e_{i}\right)$, then $x_{1}, \ldots, x_{s}$ is a system of 
generators of $M$. Let $v_{i}=v_{\mathscr{F}}\left(x_{i}\right)$ and $\mathscr{F}_{p} A^{s}=\oplus_{i=1}^{s} \mathrm{n}^{p-v_{i}} e_{i}$; then $\operatorname{gr}_{\mathscr{F}}\left(A^{s}\right)=$ $\bigoplus_{i=1}^{s} \operatorname{gr}_{\mathscr{F}}(A)\left(-v_{i}\right)$ and since $\mathrm{n}^{p-v_{i}}\left(\mathscr{F}_{v_{i}} M\right) \subseteq \mathscr{F}_{p} M, \varphi$ is a morphism of filtered modules such that $\operatorname{gr}(\varphi)\left(e_{i}^{*}\right)=x_{i}^{*}$. Also, $\varphi$ is strict if and only if $\mathscr{F}_{p} M=$ $\sum_{i=1}^{n} \mathfrak{n}^{p-v_{i}} x_{i}$ for all $p \geqslant 0$. It follows that $x_{1}, \ldots, x_{n}$ is an $\mathscr{F}$-standard base of $M$ if and only if $\operatorname{gr}(\varphi)$ is surjective, which is equivalent to saying that $\varphi$ is a strict morphism or that $(\operatorname{Ker} \varphi)^{*}=\operatorname{Ker}(\operatorname{gr}(\varphi))$.

(1.6) If $M$ is a filtered $A$-module, we denote by depth $\operatorname{gr}_{\mathscr{F}}(M)$ the number $\operatorname{grade}\left(\mathrm{n}^{*}, \operatorname{gr}_{\mathscr{F}}(M)\right)$ (note that $\mathrm{n}^{*}$ is the irrelevant ideal of $\operatorname{gr}_{\mathfrak{n}}(A)$ ).

If the residue field $A / \mathrm{n}$ is infinite and depth $\operatorname{gr}_{\mathscr{F}}(M)>0$, then we can find an element $y \in \mathfrak{n}, y \notin \mathfrak{n}^{2}$, such that $y^{*}$ is $\operatorname{gr}_{\mathscr{F}}(M)$-regular.

(1.7) Let $y$ be an element in $\mathfrak{n}, y \notin \mathfrak{n}^{2}$; if we consider the map $M \stackrel{f}{\rightarrow} M$ given by $f(m)=y m$ and define $\mathscr{G}_{p} M=\mathscr{F}_{p-1} M$ for $p \geqslant 0\left(\mathscr{F}_{-1} M=M\right)$, then $f$ is a morphism of filtered modules from $(M, \mathscr{G})$ to $(M, \mathscr{F})$ such that $\operatorname{gr}(f): \operatorname{gr}_{\mathscr{G}}(M) \rightarrow$ $\operatorname{gr}_{\mathscr{F}}(M)$ is the multiplication by $y^{*}$. Hence $y^{*}$ is $\operatorname{gr}_{\mathscr{F}}(M)$-regular if and only if $f$ is strict and $y$ is $M$-regular.

On the other hand, if we consider on $y M$ the induced filtration, we get an exact sequence of filtered modules $(M, \mathscr{G}) \stackrel{f}{\rightarrow} y M \rightarrow 0$. Thus $f$ is strict if and only if $(y M)^{*}=y^{*} \operatorname{gr}_{\mathscr{G}}(M)=y^{*} \operatorname{gr}_{\mathscr{F}}(M)$; since $\operatorname{gr}_{\mathscr{F}}(M / y M)=\operatorname{gr}_{\mathscr{F}}(M) /(y M)^{*}$, we get that $f$ is strict if and only if $\operatorname{gr}_{\mathscr{F}}(M / y M)=\operatorname{gr}_{\mathscr{F}}(M) / y^{*} \operatorname{gr}_{\mathscr{F}}(M)$. In particular, the last equality holds if $y^{*}$ is $\operatorname{gr}_{\mathscr{F}}(M)$-regular.

The proofs of all these facts are standard or can be found in $[9, \S 1]$.

2. The main theorem. Let $(A, \mathfrak{n})$ be a regular local ring with an infinite residue field $k=A / \mathfrak{n}$. If $I \subseteq \mathfrak{n}^{2}$ is an ideal in $A$ such that $R=A / I$ is a local ring of depth $d$ and maximal ideal $\mathfrak{m}$, we let $S_{R}(\mathfrak{m})$, or simply $S(\mathfrak{m})$, be the symmetric algebra of $\mathfrak{m}$ over $R$, and $\mathscr{M}=\left(\mathfrak{m}, S(\mathfrak{m})_{+}\right)$is its irrelevant ideal.

Further, let $\mathscr{R}_{A}(\mathfrak{n})=\oplus_{p \geqslant 0} \mathfrak{n}^{p}=A[\mathfrak{n} T]$ be the Rees algebra of $\mathfrak{n}$ over $A$, and $\mathscr{N}=\left(\mathrm{n}, \mathscr{R}_{A}(\mathrm{n})_{+}\right)$is its irrelevant ideal.

In the following, if $M$ is a module over a commutative ring $P$ and $x \in M$, then $\tilde{x}$ denotes the corresponding 1-form of $x$ in $S_{P}(M)$. If $N$ is a submodule of $M, \tilde{N}$ denotes the ideal of $S_{P}(M)$ generated by $\tilde{x}, x \in N$.

LemMa (2.1). Let $I \subseteq J$ be ideals of a commutative ring $P$.

(a) $S_{P / I}(J / I)$ is isomorphic to $S_{P}(J) /(I, \tilde{I})$.

(b) We have an exact sequence of $S_{P}(J)$-modules

$$
0 \rightarrow I \rightarrow S_{P}(J) / \tilde{I} \rightarrow S_{P / I}(J / I) \rightarrow 0,
$$

where $I$ is considered to be an $S_{P}(J)$-module concentrated in degree 0 .

(c) $\left[S_{P / I}(J / I)\right]_{+}=\left[S_{P}(J) / \tilde{I}\right]_{+}$.

Proof. The proof of (a) follows from the universal properties of the symmetric algebra (see [11]), while (b) and (c) are trivial consequences of (a).

COROLlARY (2.2). With the above assumptions and notations we have

$$
S_{R}(\mathrm{~m})=\bigoplus_{p \geqslant 0}\left(\mathfrak{n}^{p} / I \mathfrak{n}^{p-1}\right) \quad\left(\text { here } \mathrm{n}^{-1}=\mathfrak{n}^{0}=A\right) .
$$


Proof. Since $n$ is generated by a regular sequence, we have $\mathscr{R}_{A}(\mathrm{n})=S_{A}(\mathrm{n})$; hence the conclusion follows by the above lemma.

REMARK. The above result shows that $S(\mathrm{~m})$ has a natural structure of a graded module over $\mathscr{R}_{A}(\mathfrak{n})$ : If $a \in \mathfrak{n}$ and $\alpha=\bar{b} \in \mathfrak{n}^{p} / I \mathfrak{n}^{p-1}$, then $(a T) \alpha=\overline{a b} \in$ $\mathfrak{n}^{p+1} / I \mathfrak{n}^{p}$. Of course, this means that if $x=\bar{a} \in R=A / I$, then $(a T) \alpha=\tilde{x} \alpha$.

Let us consider the associated graded module $\operatorname{gr}_{\mathfrak{n}}(I)$; then $\operatorname{gr}_{\mathfrak{n}}(I)$ has a natural structure of a graded $\operatorname{gr}_{\mathfrak{n}}(A)$-module. Since $\operatorname{gr}_{\mathfrak{n}}(A)=\mathscr{R}_{A}(\mathfrak{n}) \otimes_{A}(A / \mathfrak{n}), \operatorname{gr}_{\mathfrak{n}}(I)$ can be considered also as a graded module over $\mathscr{R}_{A}(\mathfrak{n})$. But $I \subseteq \mathfrak{n}^{2}$, hence we have inclusions $I \mathfrak{n}^{p} / I \mathfrak{n}^{p+1} \subseteq \mathfrak{n}^{p+2} / I \mathfrak{n}^{p+1} ;$ thus we get a canonical embedding $\operatorname{gr}_{\mathrm{n}}(I)(-2) \subseteq S(\mathrm{~m})$.

In the following we shall consider the $\mathscr{N}$-depth of these algebras and denote it simply by depth. It is clear that grade $\left(\mathscr{N}, \operatorname{gr}_{\mathfrak{n}}(I)\right)=\operatorname{grade}\left(\mathfrak{n}^{*}, \operatorname{gr}_{\mathfrak{n}}(I)\right)$.

Proposition (2.3). (a) If $k \geqslant 0$ then depth $S(\mathrm{~m})_{\geqslant k}>0$ if and only if $I \mathrm{n}^{t}: \mathfrak{n}=$ $I \mathrm{n}^{t-1}$ for every $t \geqslant k$.

(b) depth $\operatorname{gr}_{\mathfrak{n}}(I)>0$ if and only if $I \mathfrak{n}^{t} \cap\left(I \mathfrak{n}^{t+2}: \mathfrak{n}\right)=I \mathfrak{n}^{t+1}$ for every $t \geqslant 0$.

(c) depth $S(\mathfrak{m})_{+}>0$ if and only if $I \mathfrak{n}: \mathfrak{n}=I$ and depth $\operatorname{gr}_{\mathfrak{n}}(I)>0$.

(d) depth $S(\mathrm{~m})>0$ if and only if depth $R>0$ and depth $\mathrm{gr}_{\mathfrak{n}}(I)>0$.

(e) If depth $\operatorname{gr}_{n}(I)>0$ and for some $k \geqslant 0$ we have $I \mathrm{n}^{k}: \mathrm{n} \subseteq I$, then

$$
\operatorname{depth} S(\mathrm{~m})_{\geqslant k}>0 \text {. }
$$

Proof. (a) If for some $t \geqslant k$ there exists $a \in I \mathrm{n}^{t}: \mathfrak{n}, a \notin I \mathrm{n}^{t-1}$, then $a \mathfrak{n} \subseteq I \mathrm{n}^{t}$ $\subseteq \mathrm{n}^{t+1}$; hence $a \in \mathfrak{n}^{t}$; thus $\bar{a} \in \mathfrak{n}^{t} / I \mathfrak{n}^{t-1}$ is a nonzero element in $S(\mathfrak{m})_{\geqslant k}$. Now for all $s \geqslant 1$ we have $a \mathfrak{n}^{s} \subseteq I \mathfrak{n}^{t+s-1}$; hence $\mathscr{N}=0: \bar{a}$, a contradiction to $\operatorname{depth} S(\mathrm{~m}) \geqslant k>0$.

Conversely, if depth $S(\mathrm{~m})_{\geqslant k}=0$, then for some $t \geqslant k$ and some $a \in \mathfrak{n}^{t}, a \notin$ $I \mathrm{n}^{t-1}$, we have $\mathcal{N}=0: \bar{a}$. Therefore, if $b \in \mathfrak{n}$, we get $(b T) \bar{a}=0 \in S(\mathfrak{m})_{t+1}$; hence $b a \in I n^{t}$, a contradiction.

(b) Same argument as in (a).

(c) If depth $S(\mathrm{~m})_{+}>0$, then $I \mathfrak{n}: \mathfrak{n}=I$, and for $t \geqslant 0$ we have $I \mathfrak{n}^{t} \cap\left(I \mathfrak{n}^{t+2}: \mathfrak{n}\right)$ $=I \mathrm{n}^{t} \cap I \mathrm{n}^{t+1}=I \mathrm{n}^{t+1}$.

Conversely, we have $I \mathfrak{n}: \mathfrak{n}=I$, and if $t>1$, using induction on $t$, we get $I \mathrm{n}^{t}: \mathfrak{n} \subseteq I \mathrm{n}^{t-1}: \mathfrak{n}=I \mathrm{n}^{t-2}$; hence $I \mathrm{n}^{t}: \mathfrak{n}=I \mathrm{n}^{t-2} \cap\left(I \mathrm{n}^{t}: \mathfrak{n}\right)=I \mathfrak{n}^{t-1}(t-2 \geqslant 0)$.

(d) If depth $S(\mathrm{~m})>0$, then $I: \mathfrak{n}=I$; hence depth $R>0$, and, by (c), we also get depth $\operatorname{gr}_{\mathrm{n}}(I)>0$.

Conversely, if depth $R>0$, then $I: \mathfrak{n}=I$; hence $I \mathfrak{n}: \mathfrak{n}=I$; we apply (c) and get the conclusion.

(e) If $k=0$ or 1 , the conclusion follows by (c) and (d). Let $k \geqslant 2$ and $t \geqslant k$; then $I \mathrm{n}^{t}: \mathfrak{n} \subseteq I \mathrm{n}^{k}: \mathfrak{n} \subseteq I$. Now if $0 \leqslant j \leqslant t-2$ and $I \mathrm{n}^{t}: \mathfrak{n} \subseteq I \mathrm{n}^{j}$, then $I \mathrm{n}^{t}: \mathrm{n} \subseteq I \mathrm{n}^{j} \cap$ $\left(I \mathrm{n}^{j+2}: \mathfrak{n}\right)=I \mathfrak{n}^{i+1}$. This proves $I \mathfrak{n}^{t}: \mathfrak{n}=I \mathfrak{n}^{t-1}$, as required.

EXAMPLE. Let $(A, \mathfrak{n})$ be a regular local ring of dimension $n$ and $\mathfrak{n}=\left(a_{1}, \ldots, a_{n}\right)$. Let $X$ be an $n \times(n+1)$-matrix such that $x_{i 1}=a_{i}$ and $x_{i j} \in \mathrm{n}^{2}$ for all $i$ and for all $j \geqslant 2$. If $I$ is the ideal generated by the maximal minors of $X$, which we call $f_{1}, \ldots, f_{n+1}$, and if we assume that $f_{1}$ is part of a minimal base of $I$, then $f_{1} \notin I \mathfrak{n}$. 
But for all $i=1, \ldots, n$ we have $\sum_{j=1}^{n+1} x_{i j} f_{j}=0$, which implies $n f_{1} \subseteq I n^{2}$; hence $f_{1} \in I \cap\left(I n^{2}: \mathfrak{n}\right)$. By the above proposition we get depth $\operatorname{gr}_{\mathfrak{n}}(I)=0$ and also depth $S(\mathrm{~m})=0$. It is clear that we can choose $A$ and $X$ in such a way that $R=A / I$ is Cohen-Macaulay of arbitrary large dimension.

Now recall that we have a natural mapping $\lambda: S(\mathrm{~m})_{+} \rightarrow S(\mathrm{~m})$ which is called the "downgrading" homomorphism and is defined as follows: An element $b_{1} \cdots b_{t+1}$ in $S(\mathrm{~m})_{t+1}$ is mapped to the element $b_{1}\left(b_{2} \cdots b_{t+1}\right)$ in $S(\mathrm{~m})_{t}$. It is clear that it does not matter which element is pulled out of the pack; it follows that $\lambda$ is a homomorphism of $S(\mathfrak{m})$-modules. Further, it is easy to see that $\operatorname{Ker} \lambda=\operatorname{gr}_{\mathfrak{n}}(I)(-2)$ and $\lambda\left(S(\mathfrak{m})_{+}\right)=\mathfrak{m} S(\mathfrak{m})$.

LeMMA (2.4). If $k$ is a positive integer such that $k \leqslant \min \left(d, \operatorname{depth}_{\operatorname{gr}_{\mathfrak{n}}}(I)\right)$, then there exist elements $y_{1}, \ldots, y_{k} \in \mathfrak{n}$ with the following properties:

(a) $\left\{y_{1}, \ldots, y_{k}\right\}$ is a part of a regular system of parameters in $A$.

(b) $y_{1}^{*}, \ldots, y_{k}^{*}$ is a regular sequence on $\operatorname{gr}_{\mathfrak{n}}(I)$.

(c) $y_{1}, \ldots, y_{k}$ is a regular sequence modulo $I$.

Proof. Since depth $\operatorname{gr}_{\mathfrak{n}}(I)>0$, by (1.6), we can find an element $a \in \mathfrak{n}, a \notin \mathfrak{n}^{2}$, such that $a^{*}$ is $\operatorname{gr}_{n}(I)$-regular. Let $\left\{\mathfrak{p}_{1}, \ldots, \mathfrak{p}_{s}\right\}=\operatorname{Max} \operatorname{Ass}(A / I)$ and assume $a \in \mathfrak{p}_{1} \cap \cdots \cap \mathfrak{p}_{i}, a \notin \mathfrak{p}_{i+1} \cup \cdots \cup \mathfrak{p}_{s}$. Using the fact that depth $(A / I)=d>0$, we get an element $b \in \mathfrak{n}^{2} \cap \mathfrak{p}_{i+1} \cap \cdots \cap \mathfrak{p}_{s}, b \notin \mathfrak{p}_{1} \cup \cdots \cup \mathfrak{p}_{i}$; if $y=a+b$, then it is clear that $y \in \mathfrak{n}, y \notin \mathfrak{n}^{2}, y^{*}$ is $\operatorname{gr}_{\mathfrak{n}}(I)$-regular and $y$ is regular modulo $I$. Hence if $k=1$, the proof is complete; if not, we can argue by induction on $k$. Let $k>1$ and let $y_{1}$ be the element chosen as before; then

$$
\operatorname{gr}_{\mathfrak{n}}(I) / y_{1}^{*} \operatorname{gr}_{\mathfrak{n}}(I)=\operatorname{gr}_{\mathfrak{n} /\left(y_{1}\right)}\left(I / y_{1} I\right)=\operatorname{gr}_{\mathfrak{n} /\left(y_{1}\right)}\left(I+\left(y_{1}\right) /\left(y_{1}\right)\right) \text {, }
$$

where the last equality is a consequence of the fact that $y_{1}$ is a regular element modulo $I$, hence $I+\left(y_{1}\right) /\left(y_{1}\right)=I / I \cap\left(y_{1}\right)=I / y_{1} I$. We denote by - the reduction modulo $\left(y_{1}\right)$. Then $\bar{A}$ is a regular local ring, and $\bar{I}=I+\left(y_{1}\right) /\left(y_{1}\right)$ an ideal contained in $\overline{\mathfrak{n}}^{2}$ such that $\bar{A} / \bar{I}$ has depth $d-1 \geqslant k-1$; further, $\operatorname{depth}_{\mathrm{gr}_{\overline{\mathrm{n}}}}(\bar{I})=$ depth $\operatorname{gr}_{n}(I)-1 \geqslant k-1$. Hence, by the inductive hypothesis, we get elements $\bar{y}_{2}, \ldots, \bar{y}_{k} \in \bar{n}$ with all the required properties. It is clear that $y_{1}, \ldots, y_{k}$ are precisely the elements we seek.

Definition. Let $y_{1}, \ldots, y_{k}$ be elements in $\mathfrak{n}$ with properties (a)-(c) of Lemma (2.4). For $i=1, \ldots, k$ let $x_{i}=y_{i}+I \in R=A / I$. Then we say that $x_{1}, \ldots, x_{k}$ is a nice regular sequence in $R$.

It is clear that if $x_{1}, \ldots, x_{k}$ is a nice regular sequence in $R$, then $\bar{x}_{2}, \ldots, \bar{x}_{k}$ is a nice regular sequence in $R /\left(x_{1}\right)=A /\left(y_{1}\right) /\left(I+\left(y_{1}\right) /\left(y_{1}\right)\right)$.

In the following, if $x$ is a nice regular sequence in $R$, say $x=y+I$, then we denote by - the reduction modulo $(y)$. Hence, $\bar{A}=A /(y)$ is still a regular local ring, $\bar{I}=I+(y) /(y) \subseteq \overline{\mathfrak{n}}^{2}, R /(x)=\bar{A} / \bar{I}$ and $\mathfrak{m} /(x)=\overline{\mathfrak{n}} / \bar{I}$. Further, $\operatorname{gr}_{\overline{\mathfrak{n}}}(\bar{I})=$ $\operatorname{gr}_{n}(I) / y^{*} \operatorname{gr}_{n}(I)$; hence, depth $\operatorname{gr}_{\bar{n}}(\bar{I})=\operatorname{depth} \operatorname{gr}_{\mathfrak{n}}(I)-1$, and depth $R /(x)=$ $d-1$.

Proposition (2.5). If $x_{1}, \ldots, x_{k}$ is a nice regular sequence in $R$, then $\tilde{x}_{1}, \tilde{x}_{2}-$ $x_{1}, \ldots, \tilde{x}_{k}-x_{k-1}$ is a regular sequence in $S(\mathrm{~m})$. 
Proof. If $\tilde{x}_{1}$ is not a regular element in $S(m)$ and $p$ is the smallest integer such that there exists $\alpha \in S(\mathfrak{m})_{p}, \alpha \neq 0$, with $\tilde{x}_{1} \alpha=0$, then $0=\lambda\left(\tilde{x}_{1} \alpha\right)=x_{1} \alpha$, which implies $p \geqslant 1$, since $x_{1}$ is a regular element in $R$. Then we get $0=\lambda\left(\tilde{x}_{1} \alpha\right)=\tilde{x}_{1} \lambda(\alpha)$; hence $\lambda(\alpha)=0$ by the minimality of $p$. Thus, $\alpha \in \operatorname{Ker} \lambda=\operatorname{gr}_{n}(I)$ and $\tilde{x}_{1} \alpha=y_{1}^{*} \alpha$ $=0$, a contradiction.

If $k=1$, the proof is complete; if not, we can argue by induction on $k$. Let $k>1$; since $x_{1}$ is a regular element in $R$, we have an exact sequence

$$
0 \rightarrow R \rightarrow S(\mathrm{~m}) /\left(\tilde{x}_{1}\right) \rightarrow S\left(\mathrm{~m} /\left(x_{1}\right)\right) \rightarrow 0 .
$$

Let $i$ be any integer, $2 \leqslant i \leqslant k$; we know that the images of $x_{2}, \ldots, x_{k}$ in $R /\left(x_{1}\right)$ form a nice regular sequence in $R /\left(x_{1}\right)$; hence, by the inductive assumption, the elements $\bar{y}_{2} T, \bar{y}_{3} T-\bar{y}_{2}, \ldots, \bar{y}_{i} T-\bar{y}_{i-1}$ form a regular sequence on $S\left(\mathrm{~m} /\left(x_{1}\right)\right)$.

If we consider the Koszul complex with respect to the elements $y_{2} T-y_{1}$, $y_{3} T-y_{2}, \ldots, y_{i} T-y_{i-1}$, from the above exact sequence we get an exact sequence

$$
\cdots \rightarrow H_{1}(\underline{y} ; R) \rightarrow H_{1}\left(\underline{y} ; S(\mathrm{~m}) /\left(\tilde{x}_{1}\right)\right) \rightarrow H_{1}\left(\underline{y} ; S\left(\mathrm{~m} /\left(x_{1}\right)\right)\right) \rightarrow \cdots .
$$

Since $R$ is considered as a graded module concentrated in degree 0 and $R /\left(x_{1}\right)=$ $\left(A /\left(y_{1}\right)\right) /\left(I+\left(y_{1}\right) /\left(y_{1}\right)\right)$, the action of $y_{2} T-y_{1}, \ldots, y_{i} T-y_{i-1}$ on $R$ (respectively on $S\left(\mathrm{~m} /\left(x_{1}\right)\right)$ ) is the same as the action of $y_{1}, \ldots, y_{i-1}$ (respectively $\bar{y}_{2} T$, $\left.\bar{y}_{3} T-\bar{y}_{2}, \ldots, \bar{y}_{i} T-\bar{y}_{i-1}\right)$. This proves that $H_{1}(y ; R)=H_{1}\left(y ; S\left(\mathrm{~m} /\left(x_{1}\right)\right)\right)=0$; hence $H_{1}\left(y ; S(m) /\left(\tilde{x}_{1}\right)\right)=0$. Since this is true for any $i=2, \ldots, k$, we conclude that $y_{2} T-y_{1}, \ldots, y_{k} T-y_{k-1}$ is a regular sequence on $S(\mathfrak{m}) /\left(\tilde{x}_{1}\right)$; hence $\tilde{x}_{1}$, $\tilde{x}_{2}-x_{1}, \ldots, \tilde{x}_{k}-x_{k-1}$ is a regular sequence in $S(\mathrm{~m})$.

REMARK. (1) We can show that the converse of (2.5) holds as well, but since we do not use this fact here, we skip the proof.

(2) Even in the case $d>0$ it can happen that $y^{*}$ is regular on $\operatorname{gr}_{11}(I)$, but $y$ is not regular modulo $I$. Let $A=k \llbracket x, y, z \rrbracket$ and $I=\left(y z-x^{5}, x^{2} z-y^{2}\right)$; then it is clear that $\operatorname{gr}_{n}(I)=A^{2} /\left(y^{2}, y z\right) A$; hence $x$ is $\operatorname{gr}_{n}(I)$-regular, but $x$ is a zero divisor modulo $I$.

In the following, if $x_{1}, \ldots, x_{k}$ are elements in $\mathrm{m}$, we set for every $i=1, \ldots, k$, $J_{i}=\left(\tilde{x}_{1}, \tilde{x}_{2}-x_{1}, \ldots, \tilde{x}_{i}-x_{i-1}\right)$ and $J=J_{k}$. Further, we let $\mathfrak{a}=\left(x_{1}, \ldots, x_{k}\right)$; hence $\tilde{\mathrm{a}}=\left(\tilde{x}_{1}, \ldots, \tilde{x}_{k}\right)$.

The next results show some other relevant properties of a nice regular sequence.

Lemma (2.6). If $x_{1}, \ldots, x_{k}$ are elements in $\mathrm{m}$, then for every $i=1, \ldots, k$ we have

(a) $x_{i} S(\mathrm{~m})_{\geqslant i} \subseteq J_{i}$.

(b) $x_{i} S(\mathrm{~m}){ }_{\geqslant i-1} \subseteq J_{i}+x_{1} R$.

(c) $\tilde{x}_{i} S(\mathrm{~m}) \geqslant i-1 \subseteq J_{i}$.

(d) If $\alpha \in J$, then $\alpha=c+\beta$ with $c \in R, \beta \in \tilde{a}$.

(e) $J \cap S(\mathrm{~m})_{\geqslant k}=\tilde{\mathrm{a}} \geqslant k$.

Proof. (a) For every $\alpha \in S(\mathfrak{m})_{+}$we have $\alpha x_{i}=\lambda(\alpha) \tilde{x}_{i}$; hence if $i=1, x_{1} \alpha \in J_{1}$; if $i>1, x_{i} \alpha \equiv x_{i-1} \lambda(\alpha) \bmod J_{i}$, and we can conclude by induction on $i$.

(b) If $i=1$ and $\alpha \in S(\mathrm{~m})$ we have, by (a), $x_{1} \alpha \equiv x_{1} \alpha_{0} \bmod J_{1}$, where $\alpha_{0}$ is the component of degree 0 in $\alpha$; hence $\alpha_{0} \in R$. If $i>1$ and $\alpha \in S(\mathrm{~m})_{\geqslant i-1}$, then $x_{i} \alpha=\tilde{x}_{i} \lambda(\alpha) \equiv x_{i-1} \lambda(\alpha) \bmod J_{i}$, and we can conclude by induction on $i$. 
(c) We may assume $i>1$ and $\alpha \in S(\mathrm{~m})_{\geqslant i-1}$; then $\tilde{x}_{i} \alpha \equiv x_{i-1} \alpha \bmod J_{i}$, and we conclude by (a).

(d) If $\alpha \in J$, the component of degree $p \geqslant 1$ in $\alpha$ can be written

$$
\alpha_{p}=\sum_{i=1}^{k} \beta_{i} \tilde{x}_{i}+\sum_{i=1}^{k-1} \gamma_{i} x_{i}
$$

with $\beta_{i} \in S(\mathrm{~m})_{p-1}, \gamma_{i} \in S(\mathrm{~m})_{p}$. Since $\gamma_{i} x_{i}=\tilde{x}_{i} \lambda\left(\gamma_{i}\right) \in \tilde{\mathrm{a}}$, the result is proved.

(e) Using (d) we get $J \cap S(\mathrm{~m})_{\geqslant k} \subseteq \tilde{\mathfrak{a}}_{\geqslant k}$. On the other hand, if $\alpha \in \tilde{\mathrm{a}}_{t}$ for some $t \geqslant k$, then $\alpha=\sum_{i=1}^{k} \beta_{i} \tilde{x}_{i}$ with $\beta_{i} \in S(\mathfrak{m})_{t-1}$; hence $\alpha \in J$, by (c).

Proposition (2.7). If $x_{1}, \ldots, x_{k}$ is a nice regular sequence in $R$, then we have $J_{i}: x_{1}=\left(x_{1}, \ldots, x_{k-1}\right)$.

Proof. We prove by induction on $i$ that $J_{i}: x_{R}=\left(x_{1}, \ldots, x_{i-1}\right)$. If $c x_{1} \in J_{1}$, then $c x_{1}=0$; hence $c=0$. Let $i>1$ and $c x_{1} \stackrel{R}{\in} J_{i}$; then for some $\alpha \in S(\mathrm{~m})$ we have $\alpha\left(\tilde{x}_{i}-x_{i-1}\right) \in\left(x_{1}, \tilde{x}_{1}, \tilde{x}_{2}, \tilde{x}_{3}-x_{2}, \ldots, \tilde{x}_{i-1}-x_{i-2}\right)$. Since the images of $x_{2}, \ldots, x_{k}$ in $R /\left(x_{1}\right)$ form a nice regular sequence, the elements $\tilde{x}_{2}, \tilde{x}_{3}-x_{2}, \ldots, \tilde{x}_{k}$ $-x_{k-1}$ form a regular sequence modulo $\left(x_{1}, \tilde{x}_{1}\right)$, by Proposition (2.5). It follows that $\alpha \in\left(x_{1}, \tilde{x}_{1}, \tilde{x}_{2}, \tilde{x}_{3}-x_{2}, \ldots, \tilde{x}_{i-1}-x_{i-2}\right)=J_{i-1}+x_{1} R$; hence $\alpha=x_{1} b+\beta$ with $b \in R, \beta \in J_{i-1}$. Now we have $c x_{1}-\left(x_{1} b+\beta\right)\left(\tilde{x}_{i}-x_{i-1}\right) \in J_{i-1}$; thus $c x_{1}$ $+b x_{1} x_{i-1} \in J_{i-1}$. By the inductive assumption we get $c+b x_{i-1} \in\left(x_{1}, \ldots, x_{i-2}\right)$; hence $c \in\left(x_{1}, \ldots, x_{i-1}\right)$.

Proposition (2.8). Let $d \geqslant 1$ and depth $\operatorname{gr}_{\mathfrak{n}}(I)=d+1$. If $x_{1}, \ldots, x_{d}$ is a nice regular sequence in $R$, then $J \underset{S(\mathrm{~m})}{:} \mathscr{M} \subseteq J+S(\mathrm{~m}) \geqslant d$.

Proof. Induction on $d$, the case $d=1$ being a consequence of the fact that $x_{1}$ is a regular element in $R$. Let $d>1$ and $\alpha \in S(\mathfrak{m})$ such that $\alpha \mathscr{M} \subseteq J$. We have $J \subseteq\left(x_{1}, \tilde{x}_{1}, \tilde{x}_{2}, \tilde{x}_{3}-x_{2}, \ldots, \tilde{x}_{d}-x_{d-1}\right)$; on the other hand, the images of $x_{2}, \ldots, x_{d}$ in $R /\left(x_{1}\right)$ form a nice regular sequence and depth $\operatorname{gr}_{\bar{\pi}}(\bar{I})=d=\operatorname{depth}\left(R /\left(x_{1}\right)\right)+1$. It follows, by the inductive assumption, that $\alpha \in\left(x_{1}, \tilde{x}_{1}, \tilde{x}_{2}, \tilde{x}_{3}-x_{2}, \ldots, \tilde{x}_{d}-\right.$ $\left.x_{d-1}\right)+S(\mathrm{~m})_{\geqslant d-1}$. Thus we may assume $\alpha=c x_{1}+\beta+\gamma$, where $c \in R, \beta \in$ $S(\mathrm{~m})_{d-1}$ and $\gamma \in S(\mathrm{~m})_{\geqslant d}$. Now we have $x_{d} \alpha \in J$; hence $c x_{1} x_{d}+\beta x_{d}+\gamma x_{d} \in J$. Since $\beta x_{d} \equiv x_{1} \lambda^{d-1}(\beta)$ mod $J$ and, by Lemma (2.6), $\gamma x_{d} \in J$, we get

$$
x_{1}\left(c x_{d}+\lambda^{d-1}(\beta)\right) \in J
$$

hence, by Proposition (2.7), $\lambda^{d-1}(\beta) \in\left(x_{1}, \ldots, x_{d}\right)$. Now let $L$ be the homogeneous ideal in $S(\mathrm{~m})$ generated by $x_{1}, \ldots, x_{d}, \tilde{x}_{1}, \ldots, \tilde{x}_{d}$; then $L=\mathfrak{a}+\tilde{a}$ and $\lambda^{d-1}(\beta) \in L$. For every $x \in \mathrm{m}$ we have $x \alpha, \tilde{x} \alpha \in J$; hence $x \alpha, \tilde{x} \alpha \in L$. This implies $x \beta$, $\tilde{x} \beta \in L$; therefore $\beta \in L: \mathscr{M}$. We claim that $\beta \in L$. Let ${ }^{-}$denote reduction modulo $\left(y_{1}, \ldots, y_{d}\right)$; we have $\operatorname{Ker} \lambda=\operatorname{gr}_{\bar{n}}(\bar{I}) \subseteq S_{R / \mathfrak{a}}(\mathrm{m} / \mathfrak{a})=S_{R}(\mathrm{~m}) / L$. Therefore the assumption depth $\operatorname{gr}_{\bar{n}}(\bar{I})>0$ can be rewritten as $(L: \mathscr{M}) \cap \lambda^{-1}(L) \subseteq L$. Now it is clear that $\lambda\left(L_{+}\right) \subseteq L$; hence, since $\beta \in L: \mathscr{M}$, we get $\lambda^{j}(\beta) \in L: \mathscr{M}$ for every $j=1, \ldots, d-1$. Thus we have $\lambda^{d-2}(\beta) \in(L: \mathscr{M}) \cap \lambda^{-1}(L) \subseteq L$; hence $\lambda^{d-3}(\beta) \in$ $(L: \mathscr{M}) \cap \lambda^{-1}(L) \subseteq L$, and so on. Eventually we get $\beta \in(L: \mathscr{M}) \cap \lambda^{-1}(L) \subseteq L$, and the claim is proved. 
By using Lemma (2.6)(b) and (c), we get $\beta \equiv x_{1} b \bmod J$, with $b \in R$. Hence, $\alpha \equiv x_{1} l+\gamma \bmod J$, with $l \in R, \gamma \in S(\mathrm{~m})_{\geqslant d}$. But $x_{d} \alpha \in J$; hence $x_{d} x_{1} l+x_{d} \gamma \in J$, which implies $x_{d} l \in J: x_{1}=\left(x_{1}, \ldots, x_{d-1}\right)$. Therefore $l \in\left(x_{1}, \ldots, x_{d-1}\right)$; hence $x_{1} l \in J$. Accordingly $\alpha \equiv \gamma \bmod J$, and the proposition is proved.

The next three propositions show the close connection between depth $S(\mathrm{~m})$ and depth $\operatorname{gr}_{\mathrm{n}}(I)$. This is the raison d'etre of the entire paper.

Proposition (2.9). If depth $S(\mathfrak{m})<d$, then depth $\operatorname{gr}_{\mathfrak{n}}(I)=\operatorname{depth} S(\mathfrak{m})$.

Proof. Let $g=$ depth $S(\mathrm{~m}), h=\operatorname{depth} \operatorname{gr}_{\mathfrak{n}}(I)$; if $g=0$, then, by Proposition (2.3), we get $h=0$. Induction on $g$; if $g>0$, then $h>0$; hence by Lemma (2.4), there exists $x \in \mathrm{m}$ which is a nice regular sequence in $R$. We have an exact sequence

$$
0 \rightarrow R \rightarrow S(\mathrm{~m}) /(\tilde{x}) \rightarrow S_{R /(x)}(\mathrm{m} /(x)) \rightarrow 0 .
$$

Hence, since depth $R=d>\operatorname{depth} S(\mathrm{~m}) /(\tilde{x})=g-1$, we get

$$
\operatorname{depth} S_{R /(x)}(\mathrm{m} /(x))=g-1 \text {, }
$$

while depth $R /(x)=d-1$. Using the inductive hypothesis, we get depth $\operatorname{gr}_{\bar{n}}(\bar{I})=$ $g-1$; hence depth $\operatorname{gr}_{\mathfrak{n}}(I)=g$.

Proposition (2.10). If depth $\operatorname{gr}_{\mathfrak{n}}(I)<d$, then depth $S(\mathfrak{m})=\operatorname{depth} \operatorname{gr}_{\mathfrak{n}}(I)$.

Proof. Let $g=\operatorname{depth} S(\mathrm{~m}), h=\operatorname{depth} \operatorname{gr}_{\mathfrak{n}}(I)$; if $h=0$, then $g=0$. Induction on $h$ : if $h>0$, then we can find a nice regular sequence $x$ in $R$. Hence,

$$
\operatorname{depth} \operatorname{gr}_{\overline{\mathrm{n}}}(\bar{I})=h-1<d-1=\operatorname{depth}(\bar{A} / \bar{I})
$$

by the inductive assumption this implies depth $S_{R /(x)}(\mathrm{m} /(x))=h-1$. Using again the exact sequence

$$
0 \rightarrow R \rightarrow S(\mathrm{~m}) /(\tilde{x}) \rightarrow S_{R /(x)}(\mathrm{m} /(x)) \rightarrow 0,
$$

where

$$
d=\operatorname{depth} R>h=\operatorname{depth} S_{R /(x)}(\mathrm{m} /(x))+1,
$$

we get depth $S(\mathfrak{m}) /(\tilde{x})=h-1$; hence depth $S(\mathfrak{m})=h$.

Proposition (2.11). If depth $\operatorname{gr}_{\mathfrak{n}}(I)=d$, then depth $S(\mathrm{~m})=d$.

Proof. We have exact sequences

$$
\begin{aligned}
& 0 \rightarrow \mathrm{gr}_{\mathfrak{n}}(I) \rightarrow S(\mathrm{~m})_{+} \stackrel{\lambda}{\rightarrow} \mathrm{m} S(\mathrm{~m}) \rightarrow 0, \\
& 0 \rightarrow S(\mathrm{~m})_{+} \rightarrow S(\mathrm{~m}) \rightarrow R \rightarrow 0, \\
& 0 \rightarrow \mathrm{m} S(\mathrm{~m}) \rightarrow S(\mathrm{~m}) \rightarrow S\left(\mathrm{~m} / \mathrm{m}^{2}\right) \rightarrow 0 .
\end{aligned}
$$

If depth $\operatorname{gr}_{n}(I)=d$, then, by Proposition (2.9), depth $S(\mathrm{~m}) \geqslant d$. If depth $S(\mathrm{~m})>d$, since depth $S\left(\mathrm{~m} / \mathrm{m}^{2}\right)>\operatorname{dim} R \geqslant d$, we get, using $(* * *)$, depth $\mathrm{m} S(\mathrm{~m})>d$. Using $(*)$, this implies depth $S(\mathrm{~m})_{+}=d$, and we get a contradiction using $(* *)$.

Proposition (2.12). depth $\operatorname{gr}_{\mathfrak{n}}(I) \leqslant d+1$. 
Proof. If $d=0$ and depth $\operatorname{gr}_{\mathfrak{n}}(I)>0$, let $y \in \mathfrak{n}, y \notin \mathfrak{n}^{2}$, such that $y^{*}$ is $\operatorname{gr}_{n 1}(I)$-regular; hence $\operatorname{gr}_{\mathfrak{n}}(I) / y^{*} \operatorname{gr}_{\mathfrak{n}}(I)=\operatorname{gr}_{\mathfrak{n} /(y)}(I / y I)$. Since $\operatorname{depth}(A / I)=0$, let $x \notin I$ such that $\mathfrak{n}=I: x$; then $x y \in I, x y \notin y I$ and $x y \mathfrak{n} \subseteq y I$. This proves that $\operatorname{depth}(I / y I)=0$; hence depth $\operatorname{gr}_{\mathrm{n} /(y)}(I / y I)=0$, which implies depth $\operatorname{gr}_{\mathfrak{n}}(I)=1$.

If $d>0$ and depth $\operatorname{gr}_{\mathrm{n}}(I)>0$, we can find a nice regular sequence $x$ in $R$. Then $\operatorname{depth}(R /(x))=d-1$; hence we can apply the inductive hypothesis to get

$$
\operatorname{depth} \operatorname{gr}_{\mathfrak{n}}(I)=1+\operatorname{depth}_{\operatorname{gr}_{\bar{n}}}(\bar{I}) \leqslant d+1 .
$$

REMARK. If $R$ is Cohen-Macaulay, one can prove, using a result of Huneke and Rossi (see [7]), that depth $S(\mathrm{~m}) \leqslant d+1$.

Let

$$
I=(x, y) \cap(z, w) \subseteq A=k[|x, y, z, w|],
$$

and let $R=A / I$, then depth $R=1$, but depth $S(\mathrm{~m})=3$. This example shows that the inequality depth $S(\mathfrak{m}) \leqslant \operatorname{depth} R+1$ is not true in general.

To give a complete description of the relationship between depth $S(\mathrm{~m})$ and depth $\operatorname{gr}_{\mathfrak{n}}(I)$, we need the following definition.

Definition. Let $t$ be a positive integer. The ring $R=A / I$ is said to have a $t$-strong socle if $I \mathfrak{n}^{t}: \mathfrak{n} \subseteq I$. $R$ is said to have a strong socle if it has a 1-strong socle.

TheOREM (2.13). (a) If depth $\operatorname{gr}_{\mathfrak{n}}(I) \leqslant d$, then depth $S(\mathfrak{m})=\operatorname{depth} \operatorname{gr}_{\mathfrak{n}}(I)$.

(b) If depth $\operatorname{gr}_{\mathrm{n}}(I)=d+1$, then depth $S(\mathrm{~m}) \geqslant d$.

(c) depth $S(\mathrm{~m}) \geqslant d+1$ if and only if depth $\mathrm{gr}_{\mathrm{n}}(I)=d+1$ and for any (there exists $a$ ) nice regular sequence $x_{1}, \ldots, x_{d}$ in $R$ (such that) the ring $R /\left(x_{1}, \ldots, x_{d}\right)$ has a d-strong socle.

Proof. (a) and (b) follow from Propositions (2.9)-(2.11). Further, if

$$
\text { depth } S(\mathrm{~m}) \geqslant d+1 \text {, }
$$

then depth $\operatorname{gr}_{\mathfrak{n}}(I)=d+1$. Hence, we have to prove that, if depth $\operatorname{gr}_{\mathfrak{n}}(I)=d+1$ and $x_{1}, \ldots, x_{d}$ is a nice regular sequence in $R$, then depth $S(\mathrm{~m}) \geqslant d+1$ if and only if $R /\left(x_{1}, \ldots, x_{d}\right)$ has a $d$-strong socle.

If we denote by - the reduction modulo $\left(y_{1}, \ldots, y_{d}\right)$, we have $\bar{A} / \bar{I}=$ $R /\left(x_{1}, \ldots, x_{d}\right)$; hence $R /\left(x_{1}, \ldots, x_{d}\right)$ has a $d$-strong socle if and only if $\bar{I} \overline{\mathfrak{n}}^{d}: \overline{\mathfrak{n}} \subseteq \bar{I}$. On the other hand, we know by Proposition (2.5) that $\tilde{x}_{1}, \tilde{x}_{2}-x_{1}, \ldots, \tilde{x}_{d}-x_{d-1}$ is a regular sequence in $S(\mathfrak{m})$; hence depth $S(\mathfrak{m}) \geqslant d+1$ if and only if depth $S(\mathrm{~m}) / J>0$. By Proposition (2.8), depth $S(\mathrm{~m}) / J>0$ if and only if $\operatorname{depth}\left(S(\mathrm{~m})_{\geqslant d} / J \cap S(\mathrm{~m})_{\geqslant d}\right)>0$. But using Lemma (2.6)(e) and Lemma (2.1)(c), we get

$$
S(\mathfrak{m})_{\geqslant d} / J \cap S(\mathfrak{m})_{\geqslant d}=S(\mathfrak{m})_{\geqslant d} / \tilde{a}_{\geqslant d}=(S(\mathfrak{m}) / \tilde{a})_{\geqslant d}=\left[S_{R / \mathfrak{a}}(\mathrm{m} / \mathfrak{a})\right]_{\geqslant d} .
$$

Since depth $\operatorname{gr}_{\bar{n}}(\bar{I})=1>0$, the conclusion follows by Proposition (2.3)(a) and (e).

3. How to compute $\operatorname{gr}_{\mathfrak{n}}(I)$ and its depth. As before, $(A, \mathfrak{n})$ is a regular local ring of dimension $n$ and $I \subseteq \mathfrak{n}^{2}$ an ideal in $A$ such that $R=A / I$ is a local ring of depth $d$ and maximal ideal $m$. Further, let $P$ be the polynomial ring $\operatorname{gr}_{\mathfrak{n}}(A)=k\left[x_{1}, \ldots, x_{n}\right]$.

We have the exact sequence

$$
0 \rightarrow I \rightarrow A \rightarrow A / I \rightarrow 0
$$


from which we get depth $I=d+1$ and h.d. ${ }_{A}(I)=n-d-1$. Since we want to compute depth $\operatorname{gr}_{n}(I)$, we need to compare minimal free resolutions of $I$ and of $\operatorname{gr}_{\mathfrak{n}}(I)$, respectively.

The following result, due to L. Robbiano (see [8]), is the starting point of our investigation. We insert here a proof for the sake of completeness.

In the following, if $F$ is a finitely generated free $A$-module with basis $e_{1}, \ldots, e_{s}$ and $a_{1}, \ldots, a_{s}$ are nonnegative integers, we define $\mathscr{F}_{p} F=\bigoplus_{i} \mathfrak{n}^{p-a_{i}} e_{i}$ for every $p \geqslant 0$ (as usual we let $\mathfrak{n}^{t}=A$ for $t \leqslant 0$ ). It is clear that $\mathscr{F}$ is a stable $n$-filtration and $\operatorname{gr}_{\mathscr{F}}(F)=\oplus_{i} P\left(-a_{i}\right)$. We call the above filtration a special filtration on $F$. If (F., d.) is a complex of finitely generated free $A$-modules, a special filtration on $\mathbf{F}$. is a special filtration on each $F_{i}$, that makes (F., $\partial$.) a filtered complex. If $\mathscr{F}$ is a special filtration on $\mathbf{F}$., then $\operatorname{gr}_{\mathscr{F}}(\mathbf{F}$.) is a homogeneous complex of free $P$-modules.

Theorem (3.1). Let $M$ be a filtered A-module and (G., d.) a free homogeneous $P$-resolution of $\operatorname{gr}_{\mathscr{F}}(M)$. Then we can build up an $A$-free resolution (F., .) of $M$ and $a$ special filtration $\mathscr{F}$ on it such that $\operatorname{gr}_{\mathscr{F}}(\mathbf{F})=.\mathbf{G}$.

Proof. If the tail of $\mathbf{G}$. is

$$
\cdots \rightarrow \bigoplus_{i=1}^{t} P\left(-a_{i}\right) \stackrel{d_{0}}{\rightarrow} \mathrm{gr}_{\mathscr{F}}(M) \rightarrow 0
$$

and we put $\alpha_{i}=d_{0}\left(e_{i}\right)$, then $\alpha_{i}=x_{i}^{*}$ with $x_{i} \in M$ and $a_{i}=v_{\mathscr{F}}\left(x_{i}\right)$. Define

$$
F_{0}=\bigoplus_{i=1}^{t} A e_{i}, \quad \partial_{0}\left(e_{i}\right)=x_{i}, \quad \mathscr{F}_{p} F_{0}=\bigoplus_{i=1}^{t} \mathfrak{n}^{p-a_{i}} e_{i} ;
$$

then $F_{0} \stackrel{\partial_{0}}{\rightarrow} M \rightarrow 0$ is a complex of filtered modules and $\operatorname{gr}\left(\partial_{0}\right)=d_{0}$. Thus $\partial_{0}$ is a strict morphism, and $F_{0} \stackrel{\partial_{0}}{\rightarrow} M \rightarrow 0$ is exact. Next, suppose we are given the exact sequence

$$
\bigoplus_{i=1}^{r} P\left(-d_{i}\right) \stackrel{p}{\rightarrow} \bigoplus_{i=1}^{s} P\left(-c_{i}\right) \stackrel{\mathrm{gr}(g)}{\rightarrow} \bigoplus_{i=1}^{t} P\left(-b_{i}\right),
$$

where $g: F_{j} \rightarrow F_{j-1}$ is a morphism of free $A$-modules of rank $s$ and rank $t$, respectively, which is strict with respect to the filtrations

$$
\mathscr{F}_{p} F_{j}=\bigoplus_{i=1}^{s} \mathfrak{n}^{p-c_{i}} e_{i}, \quad \mathscr{F}_{p} F_{j-1}=\bigoplus_{i=1}^{t} \mathfrak{n}^{p-b_{i}} e_{i} .
$$

Since $g$ is strict, we have by (1.4), $(\operatorname{Ker} g)^{*}=\operatorname{Ker} \operatorname{gr}(g)$. Hence, if $F_{j+1}=\bigoplus_{i=1}^{r} A e_{i}$ with filtration $\mathscr{F}_{p} F_{j+1}=\bigoplus_{i=1}^{r} \mathrm{n}^{p-d_{i}} e_{i}$, we can define a morphism $f: F_{j+1} \rightarrow F_{j}$ such that $F_{j+1} \stackrel{f}{\rightarrow} F_{j} \stackrel{g}{\rightarrow} F_{j-1}$ is a complex of filtered modules and such that $\operatorname{gr}(f)=$ $\varphi$. Hence, $f$ is a strict morphism, and $F_{j+1} \stackrel{f}{\rightarrow} F_{j} \stackrel{g}{\rightarrow} F_{j-1}$ is exact. The conclusion follows.

In the following for an $A$-module $M, \beta_{i}(M)$ will denote the $i$ th Betti number and $\chi(M)$ the Euler-Poincaré characteristic of $M$. 
Corollary (3.2). For every filtered $A$-module $M$ we have
(a) $\chi\left(\operatorname{gr}_{\mathscr{F}}(M)\right)=\chi(M)$.
(b) $\beta_{i}\left(\operatorname{gr}_{\mathscr{F}}(M)\right) \geqslant \beta_{i}(M)$ for every $i \geqslant 0$.
(c) h.d. $P\left(\operatorname{gr}_{\mathscr{F}}(M)\right) \geqslant$ h.d. $A$.
(d) depth $\operatorname{gr}_{\mathscr{F}}(M) \leqslant$ depth $M$.

In particular, if $I$ is an ideal in $A$, then depth $\operatorname{gr}_{\mathfrak{n}}(I) \leqslant d+1$. (This is a new proof of (2.12).)

It is worthy to remark that if we start with a minimal homogeneous resolution of $\operatorname{gr}_{\mathscr{F}}(M)$, then the $A$-free resolution of $M$ given in the proof of Theorem (3.1) is not necessarily minimal, and it is minimal if and only if $\beta_{i}\left(\operatorname{gr}_{\mathscr{F}}(M)\right)=\beta_{i}(M)$ for every $i \geqslant 0$.

Definition. A filtered $A$-module $M$ is said to be of homogeneous type with respect to the given filtration $\mathscr{F}$ if $\beta_{i}(M)=\beta_{i}\left(\operatorname{gr}_{\mathscr{F}}(M)\right)$ for every $i \geqslant 0$.

Thus if $M$ is of homogeneous type with respect to $\mathscr{F}$, then h.d.p $\left(\operatorname{gr}_{\mathscr{F}}(M)\right)=$ h.d. ${ }_{A}(M)$, and also depth $\operatorname{gr}_{\mathscr{F}}(M)=\operatorname{depth} M$.

When the $A$-module $M$ is of homogeneous type with respect to the n-adic filtration, then we simply say that $M$ is of homogeneous type.

ExAmples. (1) Let $k$ be a field, $J$ a homogeneous ideal in $P=k\left[x_{1}, \ldots, x_{n}\right]$. We set $A=k \llbracket x_{1}, \ldots, x_{n} \rrbracket$ or $A=k\left[x_{1}, \ldots, x_{n}\right]_{\mathscr{M}}$, where $\mathscr{M}=\left(x_{1}, \ldots, x_{n}\right)$, and let $I=J A$. Then it is clear that $\operatorname{gr}_{\mathfrak{n}}(A)=P$ and $I^{*}=J$; hence $\operatorname{gr}_{\mathfrak{n}}(A / I)=P / J$ and $A / I$ is an $A$-module of homogeneous type.

(2) Let $I$ be an ideal generated by a super-regular sequence in the regular local ring $A$. This means that $I=\left(f_{1}, \ldots, f_{r}\right)$, where $f_{1}, \ldots, f_{r}$ is a regular sequence and an $n$-standard base of $I$; equivalently, $f_{1}^{*}, \ldots, f_{r}^{*}$ is a regular sequence in $P=\operatorname{gr}_{\mathfrak{n}}(A)$ (see [14]). Then $R=A / I$ is of homogeneous type.

More examples of modules of homogeneous type can be given by using the following easy lemma.

LemMA (3.3). Let $(A, \mathfrak{n})$ be any local ring and $I$ an ideal such that $I$ has an $\mathrm{n}$-standard base $f_{1}, \ldots, f_{r}$ of equimultiple elements of degree $g$; then $\operatorname{gr}_{\mathfrak{n}}(I)(-g)=I^{*}$.

Proof. We have

$$
\begin{aligned}
\left(I^{*}\right)_{t} & =\left(I \cap \mathrm{n}^{t}\right)+\mathrm{n}^{t+1} / \mathrm{n}^{t+1}=I \cap \mathrm{n}^{t} / I \cap \mathrm{n}^{t+1} \\
& =I \mathrm{n}^{t-g} / I \mathrm{n}^{t-g+1}=\operatorname{gr}_{\mathfrak{n}}(I)_{t-g}=\left[\operatorname{gr}_{\mathfrak{n}}(I)(-g)\right]_{t} .
\end{aligned}
$$

(3) With the notations as in Example (1), let $J$ be generated by homogeneous elements of the same degree $g$. Then we have $\beta_{i}(I)=\beta_{i}(J)=\beta_{i}\left(I^{*}\right)=\beta_{i}\left(g_{n}(I)\right)$; it follows that $I$ is an $A$-module of homogeneous type.

Consider the ring $R=k \llbracket t^{19}, t^{26}, t^{34}, t^{40} \rrbracket=k \llbracket X, Y, Z, T \rrbracket / I$. One can prove that $I$ is generated by five elements which form an equimultiple $\mathfrak{n}$-standard base for $I$, but $I$ is not of homogeneous type.

We want to mention the paper of $\mathrm{M}$. Brundu [2], where some interesting results are proven on ideals which have a standard base of equimultiple elements. 
(4) Let $M$ be a finitely generated $A$-module with minimal free resolution (F., д.). We define on $\mathbf{F}$. the special filtration $\mathscr{F}_{p} F_{i}=\mathfrak{n}^{p-i} F_{i}$ for all $p$ and $i$. $M$ is said to have a linear resolution if $\operatorname{gr}_{\mathscr{F}}(\mathbf{F}$.$) is acyclic. In this case the module M$ is of homogeneous type.

Modules with linear resolution have been extensively studied (see for example [6, 13, 3, and 5]). We give a few examples here.

(a) Let $I$ be the ideal generated by the maximal minors of a generic $r \times s$ matrix $X=\left(x_{i j}\right)$ in the ring $A=k \llbracket x_{i j} \rrbracket$. Then $I^{*}$ has a minimal resolution

$$
0 \rightarrow \bigoplus P(-s) \rightarrow \cdots \rightarrow \bigoplus P(-r-1) \rightarrow \bigoplus P(-r) \rightarrow I^{*} \rightarrow 0,
$$

whence $\operatorname{gr}_{\mathrm{n}}(I)$ has a minimal resolution

$$
0 \rightarrow \bigoplus P(-s+r) \rightarrow \cdots \rightarrow \bigoplus P(-1) \rightarrow \bigoplus P \rightarrow \mathrm{gr}_{\mathfrak{n}}(I) \rightarrow 0 .
$$

This proves that $I$ has a linear resolution.

(b) Let $I$ be an ideal in $A$ such that $R=A / I$ is Cohen-Macaulay of minimal multiplicity, say $e(R)=h(I)+1$. Then J. Sally (see [12]) proved that $I$ has a standard base of equimultiple elements of degree 2 , and $I^{*}$ has a resolution

$$
0 \rightarrow \bigoplus P(-h-1) \rightarrow \cdots \rightarrow \bigoplus P(-3) \rightarrow \bigoplus P(-2) \rightarrow I^{*} \rightarrow 0,
$$

whence $\operatorname{gr}_{n}(I)$ has a minimal resolution

$$
0 \rightarrow \bigoplus P(-h+1) \rightarrow \cdots \rightarrow \bigoplus P(-1) \rightarrow \bigoplus P \rightarrow \mathrm{gr}_{\mathfrak{n}}(I) \rightarrow 0 .
$$

This proves that $I$ has a linear resolution.

Next we want to produce some more examples of ideals which are of homogeneous type. We start with the following easy result.

Proposition (3.4). If the ideal I can be generated by two elements, then it is of homogeneous type.

Proof. If $I$ is principal, then $I \simeq A$; hence $\operatorname{gr}_{n}(I)=\operatorname{gr}_{n}(A)=P$. If $I$ is minimally generated by $x$ and $y$, we can write $x=d a, y=d b$ with $(a, b)=1$. Hence, we have a minimal free resolution $0 \rightarrow A \stackrel{g}{\rightarrow} A^{2} \stackrel{f}{\rightarrow} I \rightarrow 0$. Let $\mathscr{F}_{p} A^{2}=\oplus \mathfrak{n}^{p} e_{i}$, $\alpha=(b,-a)$ and $v=v_{\mathscr{F}}(\alpha)$; if we let $\mathscr{F}_{p} A=\mathfrak{n}^{p-v} A$, it is clear that $f$ and $g$ are strict morphisms; hence the conclusion follows.

If we pass to consider ideals with more than two generators, then they are not necessarily of homogeneous type. More, we have seen in the example following Proposition (2.3) that it is possible that depth $\operatorname{gr}_{\mathfrak{n}}(I)=0$.

However, if we restrict ourselves to an ideal $I$ generated by a super-regular sequence, then we can prove that $I$ is of homogeneous type; hence depth $\operatorname{gr}_{\mathfrak{n}}(I)=$ $d+1$.

We need the following splitting result for $\operatorname{gr}_{\mathfrak{n}}(I)$, where, if $K$ is an ideal of a local ring $(A, \mathfrak{n})$, we put $v(K)=\min _{f \in K}\left\{v_{\mathfrak{n}}(f)\right\}$.

THEOREM (3.5). Let $(A, \mathfrak{n})$ be any local ring and $I$ an ideal in $A$ such that $I=J+K$ for some ideals $J$ and $K$. If $J \cap \mathfrak{n}^{t} \subseteq J \mathfrak{n}^{t-v(K)+1}$ for all $t \geqslant 0$, then we have

$$
\operatorname{gr}_{n}(I)=\operatorname{gr}_{n}(J) \oplus \operatorname{gr}_{\mathfrak{n}}(I / J)
$$


Proof. We have

$$
I \|^{p} / I \mathfrak{n}^{p+1}=\left(J \mathfrak{n}^{p}+I \mathfrak{n}^{p+1} / I \mathfrak{n}^{p+1}\right)+\left(K \mathfrak{n}^{p}+I \mathfrak{n}^{p+1} / I \mathfrak{n}^{p+1}\right),
$$

and this is a direct sum if we prove that

$$
\left(J n^{p}+I n^{p+1}\right) \cap\left(K \mathfrak{n}^{p}+I \mathfrak{n}^{p+1}\right)=I \mathfrak{n}^{p+1}+K \mathfrak{n}^{p} \cap\left(J \mathfrak{n}^{p}+I \mathfrak{n}^{p+1}\right) \subseteq I n^{p+1} \text {, }
$$

or, which is the same,

$$
K \mathfrak{n}^{p} \cap\left(J n^{p}+I n^{p+1}\right) \subseteq I n^{p+1}
$$

We have

$$
\begin{aligned}
K \mathfrak{n}^{p} \cap\left(J \mathfrak{n}^{p}+I \mathfrak{n}^{p+1}\right) & =K \mathfrak{n}^{p} \cap\left(J \mathfrak{n}^{p}+K \mathfrak{n}^{p+1}+J \mathfrak{n}^{p+1}\right) \\
& =K \mathfrak{n}^{p+1}+K \mathfrak{n}^{p} \cap J \mathfrak{n}^{p}
\end{aligned}
$$

hence the claim follows if we prove $K n^{p} \cap J \subseteq J n^{p+1}$. But we have

$$
K \mathfrak{n}^{p} \cap J \subseteq J \cap \mathfrak{n}^{p+v(K)} \subseteq J \mathfrak{n}^{p+1},
$$

as wanted.

Now we have

$$
\left(J_{\mathfrak{n}^{p}}+I \mathfrak{n}^{p+1} / I \mathfrak{n}^{p+1}\right)=J \mathfrak{n}^{p} / J \mathfrak{n}^{p} \cap I \mathfrak{n}^{p+1}=J \mathfrak{n}^{p} / J \mathfrak{n}^{p+1} .
$$

On the other hand,

$$
\begin{aligned}
\left(K \mathfrak{n}^{p}+I \mathfrak{n}^{p+1} / I \mathfrak{n}^{p+1}\right) & =K \mathfrak{n}^{p} / K \mathfrak{n}^{p} \cap I \mathfrak{n}^{p+1} \\
& =K \mathfrak{n}^{p} / K \mathfrak{n}^{p+1}+\left(K \mathfrak{n}^{p} \cap J \mathfrak{n}^{p+1}\right) \\
& =K \mathfrak{n}^{p} / K \mathfrak{n}^{p+1}+\left(K \mathfrak{n}^{p} \cap J\right)=\left[\operatorname{gr}_{\mathfrak{n}}(I / J)\right]_{p} .
\end{aligned}
$$

REMARK. The condition $J \cap \mathfrak{n}^{t} \subseteq J \mathfrak{n}^{t-v(K)+1}$ is satisfied if, for example, $J$ has an $\mathrm{n}$-standard base $f_{1}, \ldots, f_{r}$ such that $v\left(f_{i}\right)<v(K)$ for all $i$. In fact we have

$$
\begin{aligned}
J \cap \mathfrak{n}^{t} & =\sum_{i=1}^{r} \mathfrak{n}^{t-v\left(f_{i}\right)} f_{i} \\
& \subseteq \sum_{i=1}^{r} \mathfrak{n}^{t-v(K)+1} f_{i} \subseteq J \mathfrak{n}^{t-v(K)+1} .
\end{aligned}
$$

THEOREM (3.6). If the ideal I is generated by a super-regular sequence, then I is of homogeneous type.

Proof. Let $I=\left(f_{1}, \ldots, f_{r}\right)$ with $f_{1}^{*}, \ldots, f_{r}^{*}$ a regular sequence in $P$. If $r=1$, we can apply Proposition (3.4) to get the conclusion. Let $r>1$ and assume that $v_{1}=v_{2}=\cdots=v_{k}>v_{k+1} \geqslant \cdots \geqslant v_{r}$, where $v_{i}=v_{n}\left(f_{i}\right)$. Further, let $J=$ $\left(f_{k+1}, \ldots, f_{r}\right), K=\left(f_{1}, \ldots, f_{k}\right)$; then, by [14], $f_{k+1}, \ldots, f_{r}$ is an $n$-standard base of $J$; hence we get, by Theorem (3.5), $\operatorname{gr}_{\mathfrak{n}}(I)=\operatorname{gr}_{\mathfrak{n}}(J) \oplus \operatorname{gr}_{\mathfrak{n}}(I / J)$. We claim that $\bar{f}_{1}, \ldots, \bar{f}_{k}$ is an $\mathfrak{n} / J$-standard base of $I / J$. By [9, Theorem (2.2) and Corollary (2.3)], it is enough to prove that $v_{i}=v_{\mathrm{n} / J}\left(\bar{f}_{i}\right)$ for $i=1, \ldots, k$. But if we put $v=v_{i}$ and assume $f_{i} \in \mathrm{n}^{v+1}+J$, then $f_{i}=g+c$ with $g \in \mathfrak{n}^{v+1}, c \in J$; this implies $f_{i}-c \in$ $n^{\prime+1} \cap\left(J, f_{i}\right) \subseteq f_{i} \mathfrak{n}+J$, which gives the contradiction $f_{i} \in J$.

Using the claim, we can apply Lemma (3.3) to infer that $\operatorname{gr}_{n}(I / J) \simeq(I / J)^{*} \simeq$ $I^{*} / J^{*}$. Now we must compute the Betti numbers over $P$ of $I^{*} / J^{*}$. We can consider the Koszul resolution of $P / J^{*}$ and $P / I^{*}$, respectively; it is clear that we have a 
canonical embedding which is a morphism of complexes. The associated Coker complex is a complex of free $P$-modules which gives us a resolution of $I^{*} / J^{*}$ over $P$. Further, by the inductive assumption, we have $\beta_{i}\left(\operatorname{gr}_{n}(J)\right)=\beta_{i}(J)$; hence

$$
\begin{aligned}
\beta_{i}\left(\operatorname{gr}_{\mathrm{n}}(I)\right) & =\beta_{i}\left(\operatorname{gr}_{\mathrm{n}}(J)\right)+\beta_{i}\left(I^{*} / J^{*}\right) \\
& \leqslant \beta_{i}(J)+\beta_{i}\left(I^{*}\right)-\beta_{i}\left(J^{*}\right)=\beta_{i}(I) .
\end{aligned}
$$

The conclusion follows by using Corollary (3.2).

COROLlary (3.7). If the ideal $I$ is generated by a regular sequence $f_{1}, \ldots, f_{r}$ such that $f_{1}, \ldots, f_{r-1}$ is an $\mathrm{n}$-standard base of $J=\left(f_{1}, \ldots, f_{r-1}\right)$ and $v\left(f_{i}\right)<v\left(f_{r}\right)$ for $i=1, \ldots, r-1$, then $I$ is of homogeneous type.

Proof. We have

$$
\operatorname{gr}_{\mathfrak{n}}(I)=\operatorname{gr}_{\mathfrak{n}}(J) \oplus \operatorname{gr}_{\mathfrak{n}}(I / J)=\operatorname{gr}_{\mathfrak{n}}(J) \oplus \operatorname{gr}_{\mathfrak{n}}(A / J) ;
$$

but $\beta_{i}\left(\operatorname{gr}_{11}(J)\right)=\beta_{i}(J)$; hence

$$
\begin{aligned}
\beta_{i}\left(\operatorname{gr}_{\mathrm{n}}(I)\right) & =\beta_{i}(J)+\beta_{i}\left(P / J^{*}\right) \\
& =\left(\begin{array}{c}
r-1 \\
i+1
\end{array}\right)+\left(\begin{array}{c}
r-1 \\
i
\end{array}\right)=\left(\begin{array}{c}
r \\
i+1
\end{array}\right)=\beta_{i}(I) .
\end{aligned}
$$

The following example shows that, if in the above theorem we delete the "super" condition on the regular sequence $f_{1}, \ldots, f_{t}$, not only is $I$ not of homogeneous type, but depth $\operatorname{gr}_{11}(I)$ can be zero.

Example. Let $A=k \llbracket X, Y, Z, T \rrbracket$ and $J=\left(X^{3}-Y^{7}, X^{2} Y-X T^{3}-Z^{6}\right)$; then it is easy to check that depth $\operatorname{gr}_{\mathfrak{n}}(A / J)=0$, and one can find an $\mathrm{n}$-standard base $\left(f_{1}, \ldots, f_{8}\right)$ of $J$ such that $v\left(f_{i}\right) \leqslant 16$ for $i=1, \ldots, 8$. Hence, if we consider the ideal $I=\left(X^{3}-Y^{7}, X^{2} Y-X T^{3}-Z^{6}, Z^{19}-T^{23}\right)$, then $I$ is a complete intersection ideal such that

$$
\operatorname{gr}_{n}(I)=\operatorname{gr}_{n}(J) \oplus \operatorname{gr}_{11}(I / J)=\operatorname{gr}_{n}(J) \oplus \operatorname{gr}_{n}(A / J) ;
$$

it follows that depth $\operatorname{gr}_{n}(I)=0$. The ideal $I$ is a prime ideal; this has been verified using algorithms by Buchberger, Gröbner and T. Mora, with the aid of the "SAC-2," Gröbner basis package; see [4].

We conclude this section by considering the special case of an ideal $I$ such that h.d. $I=1$.

Let us fix a minimal free resolution of $I$,

$$
0 \rightarrow A^{r} \stackrel{g}{\rightarrow} A^{r+1} \stackrel{f}{\rightarrow} I \rightarrow 0
$$

and let $M$ be the $(r+1) \times r$ matrix associated to the map $g$ with respect to the canonical bases of $A^{r}$ and $A^{r+1}$, respectively. We put $M=\left(a_{i j}\right)$ and, for $j=1, \ldots, r$, $\alpha_{j}=\left(a_{1 j}, a_{2 j}, \ldots, a_{r+1 j}\right)$; further, let $v_{j}=\min _{i} v_{\mathrm{ll}}\left(a_{i j}\right), \mathscr{F}_{p} A^{r+1}=\oplus_{i} \mathrm{n}^{p} e_{i}$ and $\mathscr{F}_{p} A^{r}=\oplus_{j} n^{p-l}, e_{j}$. Then it is clear that $f$ and $g$ are morphisms of filtered modules, and $f$ is strict. Further, the matrix associated to the map $\operatorname{gr}(g)$ is the $(r+1) \times r$ matrix $\bar{M}=\left(\bar{a}_{i j}\right)$, where $\bar{a}_{i j} \in \mathrm{n}^{\prime \prime}, / \mathrm{n}^{\prime \prime},+1$; hence $\bar{a}_{i j}=a_{i j}^{*}$ if $v\left(a_{i j}\right)=v_{j}$, and $\bar{a}_{i j}=0$ otherwise. 
Proposition (3.8). With the above assumptions and notations, let us consider the following conditions:

(a) I is of homogeneous type.

(b) depth $\operatorname{gr}_{11}(I)=d+1$.

(c) h.d. $p \operatorname{gr}_{n}(I)=1$.

(d) $g$ is a strict morphism.

(e) $\operatorname{gr}(g)$ is injective.

(f) $\operatorname{rank} \bar{M}=r$.

Then (a), (b), and (c) are equivalent, (d), (e), and (f) are equivalent, and (d) implies (a).

Proof. It is clear by definition that (d) implies (a) and (a) implies (b), while, since $\operatorname{dim} A=\operatorname{depth} I+$ h.d. ${ }_{A} I=d+2$, (b) is equivalent to (c). Further, we know that $\chi\left(\operatorname{gr}_{n}(I)\right)=\chi(I)=1$, and $\beta_{0}\left(\operatorname{gr}_{\mathfrak{n}}(I)\right)=\beta_{0}(I)=r+1$; hence, if h.d. $\operatorname{gr}_{\mathfrak{n}}(I)=1$, we get $\beta_{1}\left(\operatorname{gr}_{11}(I)\right)=r$ and $I$ is of homogeneous type. Finally, since $0 \rightarrow A^{r} \stackrel{g}{\rightarrow} A^{r+1}$ is exact, we have that $g$ is strict if and only if $\operatorname{gr}(g)$ is injective, and this, by the McCoy theorem, is equivalent to saying rank $\bar{M}=r$.

The following example shows that, in Proposition (3.8), (a) is not equivalent to (d).

Example. Let

$$
M=\left(\begin{array}{cc}
X & X \\
Y^{2} & 0 \\
0 & Z^{2}
\end{array}\right)
$$

and $I$ be the ideal generated in $A=k \llbracket X, Y, Z \rrbracket$ by the $2 \times 2$ minors of $M$, $I=\left(Y^{2} Z^{2}, X Z^{2}, X Y^{2}\right)$. The h.d. $I=1$,

$$
\bar{M}=\left(\begin{array}{cc}
X & X \\
0 & 0 \\
0 & 0
\end{array}\right) ;
$$

hence $g$ is not strict; but if $J=\left(X Z^{2}, X Y^{2}\right)$, then

$$
\operatorname{gr}_{n}(I)=\operatorname{gr}_{n}(J) \oplus \operatorname{gr}_{n}(I / J)=\operatorname{gr}_{\mathfrak{n}}(J) \oplus(P /(X)),
$$

and from this we easily get depth $\operatorname{gr}_{\mathfrak{n}}(I)=2=d+1$.

Further, if h.d. $I>1$, also the equivalence between (a) and (b) in the above proposition does not hold.

EXAmple. Let $A=k \llbracket X, Y, Z, T_{1}, \ldots, T_{d} \rrbracket$ and $I=\left(X^{3}-Y Z, X Z-Y^{2}, Z^{3}\right)$. It is clear that $I$ is a complete intersection ideal; hence $\beta_{0}(I)=3, \beta_{1}(I)=3$ and $\beta_{2}(I)=1$. If $J=\left(X^{3}-Y Z, X Z-Y^{2}\right)$, it is easy to check that $\left\{X^{3}-Y Z, X Z-\right.$ $\left.Y^{2}, X^{2} Y-Z^{2}\right\}$ is an n-standard base of $J$; hence $\operatorname{gr}_{n}(I)=\operatorname{gr}_{n}(J) \oplus \operatorname{gr}_{n}(A / J)$. But $\operatorname{gr}_{n}(A / J)=P /\left(Y Z, X Z-Y^{2}, Z^{2}\right)$, and we have $\beta_{0}\left(\operatorname{gr}_{n}(I)\right)=2+1=3$, $\beta_{1}\left(\operatorname{gr}_{11}(I)\right)=1+3=4$ and $\beta_{2}\left(\operatorname{gr}_{n}(I)\right)=0+2=2$. This proves that $I$ is not of homogeneous type, but

$$
\text { depth } \operatorname{gr}_{\mathfrak{n}}(I)=d+3-\text { h.d. } \operatorname{gr}_{\mathfrak{n}}(I)=d+1 .
$$


Now we recall that for the ideal $I$ generated in $A=k \llbracket X, Y, Z, T_{1}, \ldots, T_{d-1} \rrbracket$ by the $3 \times 3$ minors of the matrix

$$
M=\left(\begin{array}{ccc}
X & Y & Z \\
Y^{2} & 0 & 0 \\
0 & Z^{2} & 0 \\
0 & 0 & X^{2}
\end{array}\right),
$$

we have h.d. $I=1$ and depth $\operatorname{gr}_{n}(I)=0$. (See example after Proposition (2.3).)

If we consider ideals $I$ with h.d. $I=1$ and number of generators less or equal to 3 , then something more can be said. Thus let $I$ be an ideal with a minimal free resolution $0 \rightarrow A^{2} \stackrel{g}{\rightarrow} A^{3} \stackrel{f}{\rightarrow} I \rightarrow 0$ and, as before, let $M$ be the matrix associated to the map $g$. If $\bar{M}$ has rank 2 , then we have seen that $I$ is of homogeneous type. If rank $\bar{M}=1$, then we can find a row, say the first, with $\bar{a}_{11} \neq 0$ and $\bar{a}_{12} \neq 0$. We can write $a_{11}=d x, a_{12}=d y$ with $x$ and $y$ relatively prime in $A$.

Proposition (3.9). If $x^{*}$ and $y^{*}$ are relatively prime in $P$, then h.d. ${ }_{P} \operatorname{gr}_{n}(I)=2$; hence depth $\operatorname{gr}_{11}(I)=d$.

Proof. We have $v_{1}=v(d)+v(x), v_{2}=v(d)+v(y)$; hence $v_{1}+v(y)=v_{2}+$ $v(x)$ and $\bar{a}_{11}=d^{*} x^{*}, \bar{a}_{12}=d^{*} y^{*}$. Further, since rank $\bar{M}=1$ and $d^{*} \neq 0$, we have

$$
x * \bar{a}_{22}=y^{*} \bar{a}_{21} \text { and } x * \bar{a}_{32}=y^{*} \bar{a}_{31} .
$$

This implies $h_{2}=y a_{21}-x a_{22} \in \mathrm{n}^{p+1}$ and $h_{3}=y a_{31}-x a_{32} \in \mathrm{n}^{v+1}$, where $v=v_{1}$ $+v(y)=v_{2}+v(x)$. Let $\alpha_{3}=y \alpha_{1}-x \alpha_{2}$ and $v_{3}=v_{\mathscr{F}}\left(\alpha_{3}\right)$; then $\alpha_{3}=\left(0, h_{2}, h_{3}\right)$, $v_{3} \geqslant v+1$ and $\alpha_{3}^{*}=\left(0, \bar{h}_{2}, \bar{h}_{3}\right)$ with $\bar{h}_{i} \in \mathrm{n}^{n_{3}} / \mathrm{n}^{n_{3}+1}$. Now if for some $F_{i} \in P$ we have $\sum_{i=1}^{3} F_{i} \alpha_{i}^{*}=0$, then $F_{1} d^{*} x^{*}+F_{2} d^{*} y^{*}=0$; hence $F_{1}=H y^{*}, F_{2}=-H x^{*}$ for some $H \in P$. Thus

$$
H y^{*} \bar{a}_{21}-H x^{*} \bar{a}_{22}+F_{3} \bar{h}_{2}=H y^{*} \bar{a}_{31}-H x^{*} \bar{a}_{32}+F_{3} \bar{h}_{3}=0
$$

according to $(*)$, this implies $F_{3}=0$.

Thus we have proved that if $\varphi: A^{3} \rightarrow K=\operatorname{Ker} f$ is the free presentation of $K$ given by $\varphi\left(e_{i}\right)=\alpha_{i}$, then $\operatorname{Ker} \operatorname{gr}(\varphi)=\left(y^{*},-x^{*}, 0\right) P$, where we define $\mathscr{F}_{p} A^{3}=$ $\bigoplus \|^{p-"}, e_{i}$ and $\mathscr{F}_{p} K=K \cap n^{p} A^{3}$.

Now $(y,-x,-1) \in \operatorname{Ker} \varphi$, and $v(y)+v_{1}=v(x)+v_{2}<0+v_{3}$; this implies that $(y,-x,-1)^{*}=\left(y^{*},-x^{*}, 0\right)$. According to $(1.5)$ we get $\operatorname{gr}_{n}(I)=P^{3} / \sum_{i=1}^{3} \alpha_{i}^{*} P$; hence we can easily get a free $P$-resolution of $\operatorname{gr}_{n}(I)$ :

$$
0 \rightarrow P \rightarrow P^{3} \stackrel{\operatorname{gr}(\varphi)}{\rightarrow} P^{3} \rightarrow \mathrm{gr}_{11}(I) \rightarrow 0
$$

The conclusion follows.

We remark that one may apply Proposition (3.9) in each of the following situations:

(1) $a_{11}=a_{12}$.

(2) $a_{11}$ and $a_{12}$ form a super-regular sequence in $A$.

(3) $a_{11}$ and $a_{12}$ are homogeneous elements in $k\left[X_{1}, \ldots, X_{n}\right]$ and $A=$ $k \llbracket X_{1}, \ldots, X_{n} \rrbracket$ or $A=k\left[X_{1}, \ldots, X_{n}\right]_{\mathscr{M}}$ with $\mathscr{M}=\left(X_{1}, \ldots, X_{n}\right)$. 
On the other hand, the following example shows that the result is not true in general.

EXAmple. Let $A=k \llbracket X, Y, Z, T_{1}, \ldots, T_{d-1} \rrbracket$ and $I$ be the ideal generated by the $2 \times 2$ minors of the matrix

$$
M=\left(\begin{array}{cc}
X Y-Z^{3} & X^{2} \\
Y^{5} & 0 \\
0 & Z^{6}
\end{array}\right)
$$

Then $I=\left(f_{1}=X^{2} Y^{5}, f_{2}=X Y Z^{6}-Z^{9}, f_{3}=Y^{5} Z^{6}\right)$; we can apply the splitting theorem, and we get

$$
\operatorname{gr}_{\mathfrak{n}}(I)=\operatorname{gr}_{\mathfrak{n}}\left(f_{1}\right) \oplus \operatorname{gr}_{\mathfrak{n}}\left(\bar{f}_{2}, \bar{f}_{3}\right)=P \oplus \operatorname{gr}_{\mathfrak{n}}\left(\bar{f}_{2}, \bar{f}_{3}\right) .
$$

Now it is easy to check that if $g f_{2}+h f_{1} \in \mathfrak{n}^{t}$, then $g \in\left(\mathfrak{n}^{t-10}, f_{1}\right)$; hence,

$$
\begin{aligned}
\operatorname{gr}_{\mathfrak{n}}\left(\bar{f}_{2}, \bar{f}_{3}\right) & =\operatorname{gr}_{\mathfrak{n}}\left(\bar{f}_{2}\right) \oplus \operatorname{gr}_{\mathfrak{n}}\left(I /\left(f_{1}, f_{2}\right)\right) \\
& =\operatorname{gr}_{\mathfrak{n}}\left(A /\left(f_{1}\right)\right) \oplus \mathrm{gr}_{\mathfrak{n}}\left(A /\left(f_{1}, f_{2}\right): f_{3}\right) \\
& =P /\left(X^{2} Y^{5}\right) \oplus P /\left(X^{2}, X Y-Z^{3}\right)^{*} \\
& =P /\left(X^{2} Y^{5}\right) \oplus P /\left(X^{2}, X Y, X Z^{3}, Z^{6}\right) .
\end{aligned}
$$

But h.d. $P P /\left(X^{2}, X Y, X Z^{3}, Z^{6}\right)=3$; hence, h.d. $p \operatorname{gr}_{\mathfrak{n}}(I)=3$, and depth $\operatorname{gr}_{\mathfrak{n}}(I)=$ $d-1$.

4. The strong socle condition. In this section we want to investigate the $t$-strong socle condition defined in $\S 1$. As usual, $(A, \mathfrak{n})$ is a regular local ring of dimension $n$, and $I \subseteq \mathrm{n}^{2}$ an ideal such that depth $A / I=0$. If $t$ is a positive integer, we say that the ring $R=A / I$ has a $t$-strong socle if $I \mathfrak{n}^{t}: \mathfrak{n} \subseteq I$. $R$ is said to have a strong socle if it has a 1-strong socle.

It is clear that if $R$ has a $t$-strong socle then it has a $p$-strong socle for any $p \geqslant t$. Also, $R$ has a $t$-strong socle for $t \gg 0$. An upper bound for the least integer $t$ such that $R$ has a $t$-strong socle is given by the following proposition.

Let $\gamma(R)$ denote the socle of the local ring $(R, \mathrm{~m})$. Then $\gamma(R)$ is a $k$-vector space and, by the Artin-Rees lemma, we have $\gamma(R) \cap \mathrm{m}^{s}=(0)$ for $s \gg 0$. We set, by definition, $a(R)=\sup \left\{s \mid \gamma(R) \cap \mathfrak{m}^{s} \neq 0\right\}$, and, as before, $v(I)=\min _{x \in I}\left\{v_{\mathfrak{n}}(x)\right\}$.

PROPOSITION (4.1). $R$ has a $t$-strong socle for $t>a(R)-v(I)+1$.

Proof. Let $t>a(R)-v(I)+1$ and $x \in I \mathfrak{n}^{t}: \mathfrak{n}$. Then $v(x)+1 \geqslant t+v(I)>$ $a(R)+1$; hence $v(x)>a(R)$. It follows that $x \in(I: \mathfrak{n}) \cap \mathfrak{n}^{a(R)+1} \subseteq I$; where the last inclusion follows since

$$
(I: \mathfrak{n}) \cap \mathfrak{n}^{a(R)+1}+I / I \simeq \gamma(R) \cap \mathrm{m}^{a(R)+1}=0 .
$$

In general, it is not easy to determine the least integer $t$ for which $R$ has a $t$-strong socle. But if the ideal $I$ is generated by a super-regular sequence, we can give a complete answer to this question.

Let $I=\left(f_{1}, \ldots, f_{n}\right)$, where $f_{1}, \ldots, f_{n}$ is a regular sequence with $v_{i}=v_{n}\left(f_{i}\right)$. Of course, we may assume that $v_{1} \geqslant v_{2} \geqslant \cdots \geqslant v_{n} \geqslant 2$. Further, let $x_{1}, \ldots, x_{n}$ be a regular system of parameters in $A$, and let $f_{i}=\sum_{j=1}^{n} a_{i j} x_{j}, i=1, \ldots, n$. Then we 
denote by $M$ the square matrix $\left(a_{i j}\right)$ and by $d$ its determinant. It is clear that $M$ can be chosen such that $v\left(a_{i j}\right) \geqslant v_{i}-1$; hence $v(d) \geqslant \sum_{i=1}^{n} v_{i}-n$. Further, for every $j=1, \ldots, n$ we have $d x_{j}=\sum_{i=1}^{n} M_{i j} f_{i}$ where $M_{i j}$ is the cofactor of $a_{i j}$ in $M$. The following result is due to K. I. Watanabe.

Proposition (4.2). We have $d \notin I$ and $I: \mathfrak{n}=(I, d)$.

Proof. Consider the Koszul resolutions of $A / I$ and $A / \mathfrak{n}$, respectively.

$$
\begin{aligned}
& 0 \rightarrow \wedge^{n} A^{n} \rightarrow \wedge^{n-1} A^{n} \rightarrow \cdots \rightarrow \wedge A^{n} \rightarrow A \rightarrow A / I \rightarrow 0 \\
& \downarrow d \quad \downarrow \quad \downarrow\left(a_{1,}\right) \| \quad \downarrow \\
& 0 \rightarrow \wedge^{n} A^{n} \rightarrow \wedge^{n-1} A^{n} \rightarrow \cdots \rightarrow \wedge A^{n} \rightarrow A \rightarrow A / \mathfrak{n} \rightarrow 0
\end{aligned}
$$

The morphism $\operatorname{Ext}^{n}(A / \mathfrak{n}, A)=A / \mathfrak{n} \rightarrow \operatorname{Ext}^{n}(A / I, A)=A / I$ is just the multiplication by $d$. From the exact sequence $0 \rightarrow \mathrm{n} / I \rightarrow A / I \rightarrow A / \mathrm{n} \rightarrow 0$, we have that this morphism is injective; hence $d \notin I$. Since $A / I$ is Gorenstein, its socle is one-dimensional; hence $I: \mathfrak{n}=(I, d)$.

As a trivial consequence, we get a lower bound for the least integer $t$ such that $R$ has a $t$-strong socle.

In the following we let $v=\sum_{i=1}^{n} v_{i}-n$ and $m=\sum_{i=2}^{n} v_{i}-n+1$.

Proposition (4.3). If the ideal $I$ is generated by a regular sequence and $R$ has a $t$-strong socle, then $t>m$.

Proof. It is enough to show that $d n \subseteq I n^{m}$; but $d x_{j}=\sum_{i=1}^{n} M_{i j} f_{i}$, and from this the conclusion follows.

The converse of this result can be proved under special assumptions.

Proposition (4.4). Let I be generated by a regular sequence; if $n=2$ or $v_{\bar{\pi}}(\bar{d})=v$, then $R$ has an $(m+1)$-strong socle.

Proof. Let $x \in I \mathrm{n}^{m+1}: \mathfrak{n}$. Then we can write $x=\sum_{i=1}^{n} b_{i} f_{i}+c d$ for suitable $b_{i}$ and $c$ in $A$. The conclusion follows if we prove $c \in \mathfrak{n}$. For every $j=1, \ldots, n$ we have

$$
x x_{j}=\sum_{i=1}^{n}\left(b_{i} x_{j}\right) f_{i}+c\left(\sum_{i=1}^{n} M_{i j} f_{i}\right)=\sum_{i=1}^{n}\left(b_{i} x_{j}+c M_{i j}\right) f_{i} \in I \mathrm{n}^{m+1}
$$

hence

$$
\sum_{i=1}^{n}\left(b_{i} x_{j}+c M_{i j}\right) f_{i}=\sum_{i=1}^{n} r_{i j} f_{i} \quad \text { with } r_{i j} \in \mathfrak{n}^{m+1}
$$

In particular, for $j=1, \ldots, n$ we have $b_{1} x_{j}+c M_{1 j}=r_{1 j}+s_{j}$, with $s_{j} \in\left(f_{2}, \ldots, f_{n}\right)$.

Now we can write $d=\sum_{j=1}^{n} M_{1 j} a_{1 j}$; hence we get

$$
c d=\sum_{j=1}^{n} a_{1 j}\left(r_{1 j}+s_{j}-b_{1} x_{j}\right)=\sum_{j=1}^{n} a_{1 j} r_{1 j}-b_{1} f_{1}+\sum_{j=1}^{n} a_{1 j} s_{j} .
$$


Now

$$
v\left(\sum_{j=1}^{n} a_{1 j} r_{1 j}\right) \geqslant \min _{j}\left(v\left(a_{1 j}\right)+v\left(r_{1 j}\right)\right) \geqslant v_{1}-1+m+1=v+1 .
$$

Accordingly, if $c \notin \mathfrak{n}$, we get $d \in \mathrm{n}^{v+1}+I$, a contradiction to $v_{\overline{\mathrm{n}}}(\bar{d})=v$. If $n=2$, we have $m=v_{1}-1$ and $M_{12}=-a_{21}, M_{11}=a_{22}$. Thus we get

$$
\begin{aligned}
c f_{2} & =c\left(a_{21} x_{1}+a_{22} x_{2}\right)=-x_{1} c M_{12}+x_{2} c M_{11} \\
& =-x_{1}\left(r_{12}+s_{2}-b_{1} x_{2}\right)+x_{2}\left(r_{11}+s_{1}-b_{1} x_{1}\right) .
\end{aligned}
$$

Since $s_{1}, s_{2} \in\left(f_{2}\right)$, we have $c f_{2} \in \mathrm{n}^{c_{2}+1}$ if $c \notin \pi$. This is a contradiction to $v\left(f_{2}\right)=v_{2}$.

Next we consider the matrix $\bar{M}=\left(\bar{a}_{i j}\right)$ where $\bar{a}_{i j} \in \mathfrak{n}^{v_{i}-1} / \mathfrak{n}^{v_{i}}$, and we put $D=\operatorname{det} \bar{M}$. Then $D$ is a homogeneous element in $P=\operatorname{gr}_{n}(A)$ of degree $v$. As in [10] we can prove the following result.

LEMMA (4.5). The following conditions are equivalent:

(a) $D=d^{*}$.

(b) $D \neq 0$.

(c) $v(d)=v$.

Proof. We have $D=\sum_{\sigma}(-1)^{\sigma} \bar{a}_{1 \sigma_{1}} \bar{a}_{2 \sigma_{2}} \cdots \bar{a}_{n \sigma_{n}}$, where $\sigma$ runs in the set of permutations of $\{1, \ldots, n\}$. Hence, $D$ is the residue class of $d$ in $\mathfrak{n}^{v} / \mathfrak{n}^{v+1}$. Thus, $D \neq 0$ if and only if $d \notin \mathrm{n}^{v+1}$ or, which is equivalent, $v(d)=v$.

Proposition (4.6). If the ideal $I$ is generated by a super-regular sequence, then $v(d)=v(\bar{d})=v$.

Proof. We have $f_{i}^{*}=\sum_{j=1}^{n} \bar{a}_{i j} x_{j}^{*}$; hence using Proposition (4.2) we get $D \notin I^{*}=$ $\left(f_{1}^{*}, \ldots, f_{n}^{*}\right)$. This proves $D \neq 0$; hence, $D=d^{*}$ and $v(d)=v$. Further, if $d \in \mathfrak{n}^{v+1}$ $+I$, then $d \in\left(I \cap \mathfrak{n}^{v}\right)+\mathfrak{n}^{v+1}$; hence $D=d^{*} \in I^{*}$. The conclusion follows.

Corollary (4.7). Let $I$ be generated by a regular sequence, $I=\left(f_{1}, \ldots, f_{n}\right)$. If $n=2$ or $f_{1}, \ldots, f_{n}$ is a super-regular sequence, then $R$ has a $t$-strong socle if and only if $t \geqslant m+1$.

In the case $n=2$ we can even delete the condition that $f_{1}, f_{2}$ is a regular sequence.

Corollary (4.8). Let $I=(a f, a g)$, where $f$ and $g$ are relatively prime and $v(f) \geqslant v(g)=v \geqslant 1$. Then $R$ has a $t$-strong socle if and only if $t \geqslant v$.

Proof. Let $d$ be chosen as in Proposition (4.2), with respect to the regular sequence $(f, g)$. Then $d \mathfrak{n} \subseteq(f, g) \mathfrak{n}^{v-1}$; hence $a d \mathfrak{n} \subset(a f, a g) \mathfrak{n}^{v-1}$, and of course $a d \notin(a f, a g)$. This proves that a $t$-strong socle implies $t \geqslant v$.

Conversely, let $x \in I \mathfrak{n}^{v}: \mathfrak{n}$ and $y \in \mathfrak{n}$ be a regular element $\bmod (a)$ ( $y$ does exist since depth $A=2)$. Then $x y \in(a)$; hence $x=a b$ for some $b \in A$; for every $s \in \mathfrak{n}$ we have $x s=a b s \in I \mathrm{n}^{\prime \prime}$; hence $a b s=l a f+p a g, l$ and $p$ in $\mathrm{n}^{\prime \prime}$. It follows that $b s=l f+p g$ and $b \in(f, g) \mathfrak{n}^{\prime \prime}: \mathfrak{n} \subseteq(f, g)$; this implies $x \in I$ as required. 
EXAmple. Let $A=k \llbracket X, Y, Z \rrbracket$ and $f_{1}=Y^{3}, f_{2}=X Y-Z^{3}, f_{3}=X^{2}-Y^{2} Z$. It is clear that $f_{1}, f_{2}, f_{3}$ is a regular sequence with $v_{1}=3, v_{2}=2$ and $v_{3}=2$; hence $m=2$ and $v=4$. We have $d=X Y^{2} Z^{2}$ and $d n \subseteq I n^{3}$; hence $R$ does not have an $(m+1)$-strong socle.

One can prove that $R$ has a 4-strong socle as a consequence of the following easy remark.

COROLlaRY (4.9). Let I be generated by a regular sequence; then $R$ has a $t$-strong socle for $t>\sum_{i=1}^{n-1} v_{i}-n+1$.

Proof. Since $v(d) \geqslant v$, we have $\gamma(R) \cap \mathrm{m}^{v} \neq(0)$; hence $a(R) \geqslant v$. Thus, by Proposition (4.1), we get that $R$ has a $t$-strong socle for

$$
t>a(R)-v(I)+1 \geqslant v-v_{n}+1=\sum_{i=1}^{n-1} v_{i}-n+1 \text {. }
$$

Now we want to relate the $t$-strong socle condition for the ring $R$ to some property of its $A$-free minimal resolution.

If $n=1$, then it is clear that $R$ has always a strong socle. So let us assume $n>1$. We have $\operatorname{dim} A=n$ and depth $A / I=0$; hence h.d. $A / I=n$. Let (F., d.) be a minimal free resolution of $R$. If $x_{1}, \ldots, x_{n}$ is a regular system of parameters in $A$ and $I=\left(f_{1}, \ldots, f_{r}\right)$, one has isomorphisms

$$
\gamma(R) \simeq H_{n}(\underline{x} ; R) \simeq \operatorname{Tor}_{n}(k, R) \simeq F_{n} \otimes k,
$$

where $H_{n}(\underline{x} ; R)$ is the $n$th homology group of the Koszul complex generated by the elements $x_{1}, \ldots, x_{n}$ with respect to the $A$-module $R$. To describe this isomorphism explicitly, we consider the Koszul resolution (K., $\partial$.) of $k$ and the canonical isomorphisms

$$
H_{n}(\underline{x} ; R) \tilde{\leftarrow} H_{n}(\mathbf{L}) \stackrel{\sim}{\rightarrow} \operatorname{Tor}_{n}(k, R)=F_{n} \otimes k,
$$

where $\mathbf{L}$ is the total complex $\left\{L_{p}=\oplus_{j=0}^{p}\left(K_{j} \otimes F_{p-j}\right)\right\}$ with differential

$$
\begin{aligned}
& \partial_{j} \otimes 1+(-1)^{j}\left(1 \otimes d_{p-j}\right): K_{j} \otimes F_{p-j} \rightarrow\left(K_{j-1} \otimes F_{p-j}\right) \oplus\left(K_{j} \otimes F_{p-j-1}\right) . \\
& \cdots \rightarrow \quad K_{i+1} \otimes F_{n-j} \stackrel{a_{i+1} \otimes 1}{\rightarrow} K_{j} \otimes F_{n-j} \quad \stackrel{\partial_{j} \otimes 1}{\rightarrow} K_{j-1} \otimes F_{n-j} \rightarrow \cdots \\
& \downarrow 1 \otimes d_{n-i} \quad \downarrow 1 \otimes d_{n-i} \\
& \cdots \rightarrow K_{i+1} \otimes F_{n-j-1} \stackrel{a_{j+1} \otimes 1}{\rightarrow} K_{j} \otimes F_{n-j-1} \rightarrow \cdots
\end{aligned}
$$

Now let $e_{1}, \ldots, e_{n}$ be a free base of $A^{n}$, and $\varepsilon_{1}, \ldots, \varepsilon_{r}$ a free base of $F_{1}$. Then $\sigma \in \gamma(R)$ and $\tau \in F_{n} \otimes k$ correspond if for $i=0, \ldots, n$ there exist $g_{i} \in K_{i} \otimes F_{n-i}$ such that

(1) $g_{n}=a\left(e_{1} \wedge \cdots \wedge e_{n}\right)$ with $\sigma=\bar{a} \in R\left(K_{n} \otimes F_{0} \simeq K_{n}\right)$,

(2) $\tau=\bar{g}_{0} \in F_{n} / \mathfrak{n} F_{n}=F_{n} \otimes k\left(K_{0} \otimes F_{n}=F_{n}\right)$,

(3) $(-1)^{j}\left(1 \otimes d_{n-j}\right)\left(g_{j}\right)=\left(\partial_{j+1} \otimes 1\right)\left(g_{j+1}\right)$ for $j=0, \ldots, n-1$. 
LeMMA (4.10). With the above notation we have $a \in I n^{t}: n$ if and only if $g_{n-1} \in$ $n^{\prime}\left(K_{n-1} \otimes F_{1}\right)+\operatorname{Im}\left(1 \otimes d_{2}\right)$.

Proof. We have

$$
g_{n-1}=\sum_{j=1}^{n}\left(e_{1} \wedge \cdots \wedge \hat{e}_{j} \wedge \cdots \wedge e_{n}\right) \otimes\left(\sum_{i=1}^{r} b_{i j} \varepsilon_{i}\right) .
$$

Hence

$$
\begin{aligned}
\left(1 \otimes d_{1}\right)\left(g_{n-1}\right) & =\sum_{j=1}^{n}\left(\sum_{i=1}^{r} b_{i j} f_{i}\right)\left(e_{1} \wedge \cdots \wedge \hat{e}_{j} \wedge \cdots \wedge e_{n}\right)= \pm\left(\partial_{n} \otimes 1\right)\left(g_{n}\right) \\
& = \pm \sum_{j=1}^{n} a x_{j}\left(e_{1} \wedge \cdots \wedge \hat{e}_{j} \wedge \cdots \wedge e_{n}\right) .
\end{aligned}
$$

It follows that $\sum_{i=1}^{r} b_{i j} f_{i}= \pm a x_{j}$ for $j=1, \ldots, n$. Hence $a \in I n^{t}: \mathfrak{n}$ if and only if, for every $j, \sum_{i=1}^{r} b_{i j} f_{i}=\sum_{i=1}^{r} c_{i j} f_{i}$ with $c_{i j} \in \mathrm{n}^{t}$, or, which is the same,

$$
\sum_{i=1}^{r}\left(b_{i j}-c_{i j}\right) \varepsilon_{i} \in \operatorname{Ker} d_{1}=\operatorname{Im} d_{2} \text {. }
$$

This proves the lemma.

Lemma (4.11). For a given element $g \in K_{i} \otimes F_{n-i}(1 \leqslant i \leqslant n)$, the following conditions are equivalent:

(a) $g \in \mathrm{n}^{\prime}\left(K_{i} \otimes F_{n-i}\right)+\operatorname{Ker}\left(\partial_{i} \otimes 1\right)$.

(b) $\left(\partial_{i} \otimes 1\right)(g) \in \mathrm{n}^{t+1}\left(K_{i-1} \otimes F_{n-i}\right)$.

Proof. It is clear that (a) implies (b); as for the converse it is enough to prove that $\partial_{i}(h) \in \mathfrak{n}^{t+1} K_{i-1}$ implies $h \in \mathfrak{n}^{t} K_{i}+\operatorname{Ker} \partial_{i}$ for every $h \in K_{i}$. Now $\mathbf{K}$ gives a linear resolution of the $A$-module $k$. Hence if we let $\mathscr{F}_{p} K_{j}=\mathfrak{n}^{p-j} K_{j}$, then $\partial_{i}$ is a strict morphism for every $i$. This is precisely what we need to get the conclusion.

In the following, given a positive integer $t$ and a map $f: M \rightarrow N$ of $A$-modules, we say that $f$ is $t$-good if $f^{-1}\left(\mathrm{n}^{t+1} N\right) \subseteq \mathrm{n} M$.

Thus if $M$ is a free module with base $\varepsilon_{1}, \ldots, \varepsilon_{k}$ and we put $\alpha_{i}=f\left(\varepsilon_{i}\right)$, then $f$ is $t$-good if and only if for every $i=1, \ldots, k, \alpha_{i} \notin \sum_{j \neq i} A \alpha_{j}+\mathfrak{n}^{t+1} N$.

THEOREM (4.12). (a) If $d_{i}$ is 1 -good for $i \geqslant 3$ and $d_{2}$ is $t$-good, then $R$ has a $t$-strong socle.

(b) If $R$ has a t-strong socle, then $d_{n}$ is $t$-good.

Proof. (a) Let $c \in I n^{\prime}: \mathfrak{n}$; if $c \notin I$, let $\sigma=\bar{c} \in \gamma(R)$ and $\tau \in F_{n} \otimes k$ be the corresponding element. By a suitable modification of $\sum_{i=0}^{n} g_{i}$ with a boundary element, we may assume that $g_{n}=c\left(e_{1} \wedge \cdots \wedge e_{n}\right)$. By Lemma (4.10) we have $g_{n-1} \in \mathfrak{n}^{\prime}\left(K_{n-1} \otimes F_{1}\right)+\operatorname{Im}\left(1 \otimes d_{2}\right)$. Hence, we get

$$
\left(\partial_{n-1} \otimes 1\right)\left(g_{n-1}\right)=\alpha+\left(1 \otimes d_{2}\right)(\beta)
$$

with $\alpha \in \mathfrak{n}^{t+1}\left(K_{n-2} \otimes F_{1}\right)$ and $\beta \in \mathfrak{n}\left(K_{n-2} \otimes F_{2}\right)$. This implies

$$
\left(1 \otimes d_{2}\right)\left(g_{n-2}-\beta\right) \in \mathfrak{n}^{t+1}\left(K_{n-2} \otimes F_{1}\right) \text {. }
$$


Hence, if we prove that $g_{n-2} \notin \mathrm{n}\left(K_{n-2} \otimes F_{2}\right)$, we get a contradiction to the fact that $d_{2}$ is $t$-good.

But, since $\tau \neq 0$, we have $g_{0} \notin \mathfrak{n}\left(K_{0} \otimes F_{n}\right)$, and we prove by induction on $j$ that if $j<n-2$ and $g_{i} \notin \mathrm{n}\left(K_{j} \otimes F_{n-j}\right)$ then $g_{j+1} \notin \mathrm{n}\left(K_{j+1} \otimes F_{n-j-1}\right)$. Now $d_{n-j}$ is 1 -good. Hence

$$
\left(\partial_{j+1} \otimes 1\right)\left(g_{j+1}\right)= \pm\left(1 \otimes d_{n-j}\right)\left(g_{j}\right) \notin n^{2}\left(K_{j} \otimes F_{n-j}\right),
$$

which implies $g_{j+1} \notin \mathrm{n}\left(K_{j+1} \otimes F_{n-j-1}\right)$ as required.

(b) If $g_{0} \notin \mathfrak{n} F_{n}$ and $d_{n}\left(g_{0}\right) \in \mathfrak{n}^{t+1} F_{n-1}$, let $\tau=\bar{g}_{0} \in F_{n} / \mathfrak{n} F_{n}$ and $\sigma \in \gamma(R)$ be the corresponding element. We have $\left(\partial_{1} \otimes 1\right)\left(g_{1}\right) \in \mathfrak{n}^{t+1} F_{n-1}$. Hence, by Lemma (4.11), $g_{1} \in \mathfrak{n}^{t}\left(K_{1} \otimes F_{n-1}\right)+\operatorname{Im}\left(\partial_{2} \otimes 1\right)$. By a suitable modification of $\sum_{i=0}^{n} g_{i}$ with a boundary element which can have nonzero components only in degrees one and two, we may assume that $g_{1} \in \mathrm{n}^{t}\left(K_{1} \otimes F_{n-1}\right)$. Hence,

$$
\left(\partial_{2} \otimes 1\right)\left(g_{2}\right) \in \mathfrak{n}^{t+1}\left(K_{1} \otimes F_{n-2}\right),
$$

and we can apply the same argument to $g_{2}$. Eventually we get $g_{n-1} \in \mathfrak{n}^{t}\left(K_{n-1} \otimes F_{1}\right)$; by Lemma (4.10), this implies that $R$ does not have a $t$-strong socle.

REMARK. It follows from the above theorem that, if h.d. $I=1$, then $R$ has a $t$-strong socle if and only if $d_{2}$ is $t$-good. On the other hand, if $n=3$ and $I$ is the ideal generated by a regular sequence $f_{1}, f_{2}, f_{3}$ with $v\left(f_{1}\right) \geqslant v_{2}=v\left(f_{2}\right) \geqslant v_{3}=v\left(f_{3}\right)$, we have seen that, if $R$ has a $t$-strong socle, then $t \geqslant v_{2}+v_{3}-1$. But in the Koszul resolution of $R$, the map $d_{3}: A \rightarrow A^{3}$ is given by $d_{3}(1)=\left(f_{3}, f_{2}, f_{1}\right)$; hence $d_{3}$ is $v_{3}$-good. Nevertheless, $R$ does not have a $v_{3}$-strong socle.

Corollary (4.13). If $R=A / I$ is Gorenstein, then $R$ has a strong socle if and only if $I$ is a principal ideal.

Proof. If $I$ is a principal ideal, it is clear that $R$ has a strong socle. If $I$ is not principal, then $n=\operatorname{dim} A>1$. Let $(\mathbf{F}$., $d$.) be a minimal free resolution of $R$; taking Hom, we get a resolution of $\operatorname{Ext}^{n}(R, A)=R$. Hence, $d_{n}^{*}\left(F_{n-1}^{*}\right) \subseteq I \subseteq \mathrm{n}^{2}$, which implies $d_{n}\left(F_{n}\right) \subseteq \mathrm{n}^{2} F_{n-1}$. This proves that $d_{n}$ is not 1-good; hence the conclusion follows.

COROLlARY (4.14). If I has a linear resolution, then $R$ has a strong socle.

Proof. Let $\left(\mathbf{F} ., d\right.$.) be a minimal free resolution of $I$; if we put $\mathscr{F}_{p} F_{i}=\mathrm{n}^{p-i} F_{i}$, then all the $d_{j}$ are strict morphisms. Hence, if $d_{i}(\alpha) \in \mathfrak{n}^{2} F_{i-1}$, then $d_{i}(\alpha)=d_{i}(\beta)$ with $\beta \in \mathscr{F}_{i+1} F_{i}=\mathfrak{n} F_{i}$. It follows that $\alpha-\beta \in \operatorname{Ker} d_{i} \subseteq \mathfrak{n} F_{i}$; hence $\alpha \in \mathfrak{n} F_{i}$.

The converse of the above corollary does not hold. However, if h.d. $I=1$ or $I$ has an n-standard base of equimultiple elements, then we have the following complete results.

Lemma (4.15). If $R$ is an $A$-module of homogeneous type, then $\gamma(R)^{*}=\gamma\left(\operatorname{gr}_{11}(R)\right)$.

Proof. It is clear that $\gamma(R)^{*} \subseteq \gamma\left(\operatorname{gr}_{n}(R)\right)$; further, if $\gamma(R)=\left(a_{1}, \ldots, a_{t}\right)$, then $\gamma(R)^{*}=\left(a_{1}^{*}, \ldots, a_{t}^{*}\right)$. Thus, we get

$$
\operatorname{dim}_{h} \gamma(R)^{*}=\operatorname{dim}_{k} \gamma(R)=\beta_{n}(R)=\beta_{n}\left(\operatorname{gr}_{n}(R)\right)=\operatorname{dim}_{k} \gamma\left(\operatorname{gr}_{n}(R)\right) .
$$

The assertion follows. 
REMARK. If $\gamma(R)=\left(a_{1}, \ldots, a_{t}\right)$, it is clear that we have $a(R)=\max _{i}\left(v_{\mathrm{m}}\left(a_{i}\right)\right)=$ $\max _{i}\left(\operatorname{deg} a_{i}^{*}\right)$. Now if $R$ is an $A$-module of homogeneous type and $(\mathbf{F} ., d$.) is a minimal homogeneous $P$-free resolution of $\operatorname{gr}_{\mathfrak{n}}(R)$, we can use Lemma (4.15) and the canonical isomorphism $\gamma\left(\operatorname{gr}_{\mathfrak{n}}(R)\right) \simeq F_{n} \otimes k$ to get $a(R)=\max _{i}\left\{d_{n, i}\right\}-n$, where $d_{j, i}$ are the shifts in $F_{j}$.

It follows from Proposition (4.1) that if $R$ is an $A$-module of homogeneous type, then $R$ has a $t$-strong socle for $t>\max _{i}\left\{d_{n, i}\right\}-n-\min _{i}\left\{d_{1, i}\right\}+1$.

Proposition (4.16). If the ideal I is generated by an $\mathrm{n}$-standard base of equimultiple elements of degree $g$, then the following conditions are equivalent:

(1) $R$ has a strong socle and I is an A-module of homogeneous type.

(2) I has a linear resolution.

Proof. We need only prove that (1) implies (2). We know that $I^{*}=\operatorname{gr}_{\mathfrak{n}}(I)(-g)$; let $\left(\mathbf{F} ., d_{\text {.) }}\right.$ be a minimal free resolution of $I$ with a given special filtration $\mathscr{F}_{p} F_{j}=$ $\oplus_{i} n^{p-d_{11}} e_{i}$ such that $\operatorname{gr}_{\mathscr{F}}\left(\mathbf{F}\right.$.) is a homogeneous $P$-resolution of $\operatorname{gr}_{n}(I)$. Then we get a $P$-free homogeneous resolution of $\operatorname{gr}_{11}(R)$ :

$$
0 \rightarrow \bigoplus P\left(-d_{n-1, i}-g\right) \rightarrow \cdots \rightarrow \bigoplus_{i} P\left(-d_{0 i}-g\right) \rightarrow P \rightarrow \mathrm{gr}_{\mathfrak{n}}(R) \rightarrow 0 .
$$

It is clear that, for every $i$, we have $d_{0 i}+g=g$ and also by the minimality of the resolution, $d_{j i}+g>d_{j-1, i^{\prime}}+g$ for all $i, j$ and suitable $i^{\prime}$. Thus, if we prove that $d_{n-1, i}+g=g+n-1$ for every $i$, then we get $d_{j i}=j$ for every $i$ and $j$ as required.

Now, as in the above remark, we have $d_{n-1, i}+g=v_{\mathfrak{m}}\left(a_{i}\right)+n$ for some $a_{i} \in$ $\gamma(R)$; hence we need only prove that for an element $a \in \gamma(R), a \neq 0$, we have $v_{\mathrm{m}}(a)=g-1$. But if $a=\bar{x} \in R$, then $x \notin I$ and $x \mathfrak{n} \subseteq I \subseteq \mathfrak{n}^{g}$; hence $x \in \mathfrak{n}^{g-1}$ and $a \in \mathrm{m}^{g-1}$. On the other hand, if $a \in \mathrm{m}^{g}$, then $x \in \mathfrak{n}^{g}+I=\mathfrak{n}^{g}$, so $x \mathfrak{n} \subseteq I \cap$ $\mathrm{n}^{g+1}=I \mathrm{n}$, which contradicts the assumption that $R$ has a strong socle.

Finally, let $I$ be an ideal with h.d. $I=1$. As in $\$ 3$ we fix a minimal free resolution of $I$,

$$
0 \rightarrow A^{r} \stackrel{g}{\rightarrow} A^{r+1} \stackrel{f}{\rightarrow} I \rightarrow 0
$$

and we define $\mathscr{F}_{p} A^{r+1}=\bigoplus_{i} \mathrm{n}^{p} e_{i}$. This is the only special filtration on $A^{r+1}$ such that $f$ is a morphism of filtered modules; moreover, $f$ is a strict morphism.

Proposition (4.17). If h.d. $I=1$, the following conditions are equivalent:

(1) $R$ has a t-strong socle, and I is of homogeneous type.

(2) $g$ is strict with respect to a special filtration $\mathscr{F}_{p} A^{r}=\oplus_{i} \mathfrak{n}^{p-a_{i}} e_{i}$, where $a_{i} \leqslant t$ for every $i$.

Proof. If $I$ is of homogeneous type, then, by Theorem (3.1), there exist positive integers $a_{1}, \ldots, a_{r}$ such that $g$ is a strict morphism with respect to the filtration given by $\mathscr{F}_{p} A^{r}=\oplus \mathfrak{n}^{p-a_{i}} e_{i}$. Let us suppose by contradiction that $a_{i}>t$ for some $i$; since $e_{i} \in \mathscr{F}_{a_{i}} A^{r}$, we have $g\left(e_{i}\right) \in \mathscr{F}_{a_{i}} A^{r+1}=\mathfrak{n}^{a_{i}} A^{r+1} \subseteq \mathfrak{n}^{t+1} A^{r+1}$. This means that $g$ is not $t$-good, a contradiction to the fact that $R$ has a $t$-strong socle. 
Conversely, if $g(\alpha) \in \mathrm{n}^{t+1} A^{r}=\mathscr{F}_{t+1} A^{r+1}$, then

$$
\alpha \in \mathscr{F}_{t+1} A^{r}=\bigoplus \mathrm{n}^{t+1-a_{i}} e_{i} \subseteq \mathrm{n} A^{r}
$$

this proves that $g$ is $t$-good; hence $R$ has a $t$-strong socle. The conclusion follows since, if (2) holds, then $I$ is of homogeneous type.

Let $M$ be the matrix associated to the map $g$ with respect to the canonical bases; if $M=\left(a_{i j}\right)$, we let $M_{2}=\left(\bar{a}_{i j}\right)$, where $\bar{a}_{i j} \in \mathfrak{n} / \mathrm{n}^{2}$.

COROllary (4.18). If h.d. $I=1$, then the following conditions are equivalent:

(1) $R$ has a strong socle and I is of homogeneous type.

(2) I has a linear resolution.

(3) rank $M_{2}=r$.

Proof. If we let $\mathscr{F}_{p} A^{r}=\mathfrak{n}^{p-1} A^{r}$, then it is clear that $g$ is a morphism of filtered modules and $M_{2}$ is the matrix associated to $\operatorname{gr}(g)$. Also $I$ has a linear resolution if and only if $\operatorname{gr}(g)$ is injective or, which is the same, rank $M_{2}=r$. This proves that (2) and (3) are equivalent, while (1) and (2) are equivalent by Proposition (4.17).

EXAMPLES. 1. Let $I=\left(X^{3}, X^{2} Y^{2}, Y^{3}\right) \subseteq A=k \llbracket X, Y \rrbracket . I$ is generated by the $2 \times 2$ minors of the matrix

$$
M=\left(\begin{array}{cc}
Y^{2} & 0 \\
-X & Y \\
0 & -X^{2}
\end{array}\right)
$$

we have rank $M_{2}=1$, and, by Proposition (3.8), $I$ is not of homogeneous type (in this example $M_{2}=\bar{M}$ ). However, it is clear that $R$ has a strong socle.

2. Let $I=\left(X^{4}, X Y^{2}, Y^{4}\right) \subseteq A=k \llbracket X, Y \rrbracket . I$ is generated by the $2 \times 2$ minors of the matrix

$$
M=\left(\begin{array}{cc}
0 & Y^{2} \\
X & 0 \\
Y^{2} & X^{3}
\end{array}\right) ;
$$

we have rank $M_{2}=1$ and rank $\bar{M}=2$ (see Proposition (3.8)). Hence $I$ is of homogeneous type, but $R$ has not a strong socle.

5. Applications. In this section we apply the results proved before to compute depth $S(\mathrm{~m})$ for some classes of local rings $R$. As usual, $(A, \mathfrak{n})$ is a regular local ring of dimension $n$, and $I \subseteq \mathrm{n}^{2}$ an ideal such that $R=A / I$ is a local ring of depth $d$ and maximal ideal $\mathrm{m}$.

THEOREM (5.1). Let $I$ be the ideal generated by the regular sequence $f_{1}, \ldots, f_{r}$, with $v_{1} \geqslant v_{2} \geqslant \cdots \geqslant v_{r}$. If $r=2$ or $f_{1}, \ldots, f_{r}$ is a super-regular sequence, then

(a) $d \leqslant \operatorname{depth} S(\mathrm{~m}) \leqslant d+1$.

(b) depth $S(\mathrm{~m})=d+1$ if and only if $d \geqslant \sum_{i=2}^{r} v_{i}-r+2$.

Proof. By Proposition (3.4) and Theorem (3.6) we know that depth $\operatorname{gr}_{n}(I)=d+$ 1; hence the first assertion follows by Theorem (2.13). Further, depth $S(\mathrm{~m})=d+1$ if and only if there exists a nice regular sequence $x_{1}, \ldots, x_{d}$ in $R$ such that the ring $R /\left(x_{1}, \ldots, x_{d}\right)$ has a $d$-strong socle. If we can find a nice regular element $x=y+I$ $\in R$ such that $\bar{f}_{1}, \ldots, \bar{f}_{r}$ is a super-regular sequence in $A /(y)$ (respectively, $\bar{f}_{1}, \bar{f}_{2}$ is 
a regular sequence in the case $r=2)$ and $v_{i}=v_{\overline{\mathrm{n}}}\left(\bar{f}_{i}\right)$ for $i=2, \ldots, r$, then, after repeating this procedure $d$ times, we get the conclusion by Corollary (4.7).

Thus, let us first assume that $f_{1}, \ldots, f_{r}$ is a super-regular sequence. We have

$$
\text { depth } \operatorname{gr}_{n}(R)=d>0 \text {. }
$$

Hence, we can find an element $y \in \mathfrak{n}, y \notin \mathrm{n}^{2}$ such that $y^{*}$ is $\operatorname{gr}_{\mathrm{n}}(R)$-regular. We claim that $x=y+I$ is a nice regular element in $R$, or, which is the same, that $y^{*}$ is $\operatorname{gr}_{11}(I)$-regular. This is trivial if $r=1$, since, in that case, $\operatorname{gr}_{n}(I)=P$; if $r>1$, we assume $v_{1}=v_{2}=\cdots=v_{k}>v_{k+1} \geqslant \cdots \geqslant v_{r}$ and let $J=\left(f_{k+1}, \ldots, f_{r}\right)$. Then, as in the proof of Theorem (3.6), we have $\operatorname{gr}_{\mathfrak{n}}(I)=\operatorname{gr}_{\mathfrak{n}}(J) \oplus\left(I^{*} / J^{*}\right)$; thus $y^{*}$ is $\left(I^{*} / J^{*}\right)$-regular. Hence the claim follows by induction on $r$, since clearly $y^{*}$ is also $\operatorname{gr}_{n}(A / J)$-regular.

Now, since $f_{1}^{*}, \ldots, f_{r}^{*}, y^{*}$ is a regular sequence in $P$, by using Theorem (2.2) in [9], we get the conclusion if we prove that $v_{i}=v_{\bar{n}}\left(\bar{f}_{i}\right)$ for every $i$. But if $f_{i} \in \mathrm{n}^{v_{i}+1}+$ $(y)$, then $f_{i}^{*} \in\left(y^{*}\right)$, a contradiction.

Finally, let $r=2$ and $v_{1} \geqslant v_{2}$; since depth $\operatorname{gr}_{\mathfrak{n}}(A)=d+2$, we can find an element $y \in \mathfrak{n}, y \notin \mathfrak{n}^{2}$ such that $y^{*}$ and $f_{2}^{*}$ are relatively prime in $P$ and also $y$ is a regular element modulo $I$ (see the proof of Lemma (2.4)). Now it is clear that if $v_{2}<v_{1}$, then $\operatorname{gr}_{n}(I)=P \oplus P /\left(f_{2}^{*}\right)$, while, if $v_{2}=v_{1}, \operatorname{gr}_{n}(I)=P^{2} / H$, where $H$ is the cyclic $P$-module generated by $\left(f_{2}^{*},-f_{1}^{*}\right)$. In any case it is easy to check that $y^{*}$ is $\operatorname{gr}_{11}(I)$-regular. Also if $f_{2} \in \mathfrak{n}^{v_{2}+1}+(y)$, then $f_{2}^{*} \in\left(y^{*}\right)$, a contradiction. This shows that $v_{\bar{\pi}}\left(\bar{f}_{2}\right)=v_{2}$, and, as previously observed, the theorem follows.

REMARK. Let $I=(a f, a g)$, where $f$ and $g$ are relatively prime and $v(f) \geqslant v(g)$ $=v \geqslant 1$. Then depth $\operatorname{gr}_{\mathfrak{n}}(I)=d+1$ by Proposition (3.4); hence $d \leqslant \operatorname{depth} S(\mathrm{~m})$. By a slight modification of the argument used in the proof of Theorem (5.1) for the case $r=2$, one can show that it is possible to find a nice regular sequence in $R$ which does not change the valuation of $g$. Hence, we can apply Corollary (4.7), and we get that depth $S(\mathrm{~m}) \geqslant d+1$ if and only if $d \geqslant v$.

Proposition (5.2). If the ideal I has a linear resolution, then depth $S(m) \geqslant d+1$. If, moreover, $R$ is Cohen-Macaulay, then depth $S(\mathrm{~m})=d+1$.

Proof. Since $I$ is of homogeneous type, we have depth $\operatorname{gr}_{\mathfrak{n}}(I)=d+1$. Now it is clear that if $x=y+I$ is a nice regular element in $R$ and $(\mathbf{F}, \bar{d})$ is a minimal free resolution of $I$, then $(\mathbf{F} / y \mathbf{F}, \bar{d})$ is a minimal free resolution of $\bar{I}=I+(y) /(y)$. Let $\mathscr{F}$ be the special filtration on $\mathbf{F}$ such that $\operatorname{gr}_{\mathscr{F}}(\mathbf{F})$ is the minimal $P$-free resolution of $\operatorname{gr}_{n}(I)$; since $y^{*}$ is $P$-regular and also $\operatorname{gr}_{n}(I)$-regular, we get that $\operatorname{gr}_{\mathscr{F}}(\mathbf{F}) / y^{*} \operatorname{gr}_{\mathscr{F}}(\mathbf{F})$ is the minimal $P / y^{*} P$-free resolution of $\operatorname{gr}_{n}(I) / y^{*} \mathrm{gr}_{n}(I)$. But $\operatorname{gr}_{\mathscr{F}}(\mathbf{F}) / y^{*} \mathrm{gr}_{\mathscr{F}}(\mathbf{F}) \simeq$ $\operatorname{gr}_{\mathscr{F}}(\mathbf{F} / y \mathbf{F})$ and $\operatorname{gr}_{\mathfrak{n}}(I) / y^{*} \operatorname{gr}_{\mathfrak{n}}(I)=\operatorname{gr}_{n}(I / y I)=\operatorname{gr}_{\mathfrak{n}}(\bar{I})$. Hence, $\bar{I}$ has a linear resolution, and the conclusion follows by Corollary (4.14).

Proposition (5.3). Let I be an ideal of homogeneous type. Then depth $S(m) \geqslant d$. If, moreover, $I$ is generated by an n-standard base of equimultiple elements and $R$ is Cohen-Macaulay of depth $d=1$, then depth $S(\mathrm{~m})=2$ if and only if $I$ has a linear resolution. 
Proof. Since $I$ is of homogeneous type, depth $\operatorname{gr}_{n}(I)=d+1$. Hence, the first assertion follows by Theorem (2.13). As for the second one, let $I=\left(f_{1}, \ldots, f_{r}\right)$ and $y \in \mathrm{n}, y \notin \mathrm{n}^{2}$ such that $y^{*}$ is $\mathrm{gr}_{\mathrm{n}}(R)$-regular. This implies that $y$ is $R$-regular and also $v_{n 1}\left(f_{i}\right)=v_{11 /(v)}\left(\bar{f}_{i}\right)$ for every $i=1, \ldots, r$. Further, if $x=y+I$, then $v_{n / I}(x)=$ $v_{11}(y)=1$ and $x^{*}$ is $\operatorname{gr}_{\mathrm{n}}(R)$-regular. According to Theorem (2.2) in [9], this proves that $\bar{f}_{1}, \ldots, \bar{f}_{r}$ is an $\mathrm{n} /(y)$-standard base of $\bar{I}$ of equimultiple elements. We can apply Proposition (4.16) to get the conclusion.

Proposition (5.4). If $R$ is Gorenstein of depth 1 , then depth $S(\mathrm{~m})=2$ if and only if $I$ is a principal ideal.

Proof. The "if" part is clear; so let us suppose that depth $S(\mathfrak{m})=2$, and let $x=y+I$ be a nice regular element in $R$. Then $R /(x)$ is Gorenstein of depth 0 , and it has a strong socle. By Corollary (4.13) it follows that $I+(y) /(y)$ is principal. But $y$ is regular modulo $I$; hence $I$ is principal.

As in the previous sections, if $I$ is a perfect ideal of codimension 2, we fix a minimal free resolution of $I$,

$$
0 \rightarrow A^{r} \stackrel{g}{\rightarrow} A^{r+1} \stackrel{f}{\rightarrow} I \rightarrow 0
$$

and denote by $M$ the $(r+1) \times r$ matrix associated to $g$. If $M=\left(a_{i j}\right)$, we let $v_{j}=\min _{i} v\left(a_{i j}\right), \bar{M}=\left(\bar{a}_{i j}\right)$, where $\bar{a}_{i j} \in \mathfrak{n}^{v^{\prime}} / \mathfrak{n}^{v^{\prime}+1}$ and $M_{2}=\left(\tilde{a}_{i j}\right)$, where $\tilde{a}_{i j} \in$ $n / n^{2}$.

Proposition (5.5). Let I be a perfect ideal of codimension 2.

(a) If rank $\bar{M}=r$, then $d \leqslant \operatorname{depth} S(\mathrm{~m}) \leqslant d+1$.

(b) If $d=1$, then the following conditions are equivalent:

(1) depth $S(\mathrm{~m})=2$.

(2) rank $M_{2}=r$.

(3) I has a linear resolution.

Proof. By Proposition (3.8) and Theorem (2.13) we get (a). If $d=1$ and depth $S(\mathrm{~m})=2$, let $x=y+I$ be a nice regular element in $R$. Then $\bar{I}=I+$ $(y) /(y)$ is a perfect ideal of codimension 2 in $A /(y)$, and we have depth $\operatorname{gr}_{\overline{i 1}}(\bar{I})=1$. By Proposition (3.8), this implies that $\bar{I}$ is of homogeneous type; since $\bar{R}=A /(y) / \bar{I}$ has a strong socle, we get, by Corollary (4.18) a maximal minor $d$ in $M$ such that $d \notin \mathrm{n}^{r+1}+(y)$. Accordingly, $d \notin \mathrm{n}^{r+1}$, so rank $M_{2}=r$. On the other hand, it is clear that rank $M_{2}=r$ if and only if $I$ has a linear resolution. We complete the proof of (b) with the aid of Proposition (5.2).

EXAMPLES. 1. Let $I$ be the defining ideal of the monomial space curve with parametric equations $X=t^{a}, Y=t^{b}, Z=t^{c}$, where we may assume $(a, b, c)=1$. Then $I$ is a perfect ideal in $A=k \llbracket X, Y, Z \rrbracket$ of codimension 2. If $R$ has an embedding dimension 2, then depth $S(\mathrm{~m})=2$. If $R$ has an embedding dimension 3 and $I$ is a complete intersection, then depth $S(m)=1$, by Theorem (5.1). If $I$ is not 
a complete intersection, then $I$ is generated by the $2 \times 2$ minors of a matrix

$$
M=\left(\begin{array}{ll}
X^{p_{1}} & Y^{q_{1}} \\
Y^{p_{2}} & Z^{q_{2}} \\
Z^{p_{3}} & X^{q_{3}}
\end{array}\right),
$$

where $p_{i}, q_{i} \geqslant 1$. By Proposition (3.9) we get

(a) $1 \leqslant \operatorname{depth} S(\mathrm{~m}) \leqslant 2$,

and by Proposition (5.5) we get

(b) depth $S(\mathrm{~m})=2$ if and only if rank $M_{2}=2$. It is easy to see that rank $M_{2}=2$ if and only if $p_{i}=p_{j}=1$ for some $i, j \in\{1,2,3\}, i \neq j$.

2. Let $J$ be the ideal generated by the pfaffians of a $(2 g+1) \times(2 g+1)$ generic antisymmetric matrix $M=\left(X_{i j}\right)$ in $P=k\left[X_{i j}\right]$.

If $A=k\left[\left|X_{i j}\right|\right]$ and $I=J A$, then $I^{*}$ has a graded $P$-resolution

$$
0 \rightarrow \bigoplus P(-2 g-1) \rightarrow \bigoplus P(-g-1) \rightarrow \bigoplus P(-g) \rightarrow I^{*} \rightarrow 0
$$

Thus $I$ does not have a linear resolution, but $I$ and $R=A / I$ are of homogeneous type. It follows that depth $\operatorname{gr}_{\mathfrak{n}}(I)=d+1$, where

$$
\begin{aligned}
d & =\operatorname{depth}(A / I)=\operatorname{dim} A-h(I) \\
& =2 g(2 g+1) / 2-3=2 g^{2}+g-3 .
\end{aligned}
$$

If $x_{1}, \ldots, x_{d}$ is a nice regular sequence in $R$, then $\bar{R}=R /\left(x_{1}, \ldots, x_{d}\right)$ is an $\bar{A}$-module of homogeneous type. Hence we get that $\bar{R}$ has a $g$-strong socle (see the remark after Lemma (4.15)). But $d=2 g^{2}+g-3 \geqslant g$; hence depth $S(\mathrm{~m})=d+1$ $=2 g^{2}+g-2$.

3. Let $J$ be the ideal generated by the $n \times n$ minors of a generic $(n+1) \times(n+1)$ matrix $M=\left(X_{i j}\right)$ in $P=k\left[X_{i j}\right]$. If $A=k\left[\left|X_{i j}\right|\right]$ and $I=J A$, then $I^{*}=J$ has a resolution

$0 \rightarrow \bigoplus P(-2 n-2) \rightarrow \bigoplus P(-n-2) \rightarrow \bigoplus P(-n-1) \rightarrow \bigoplus P(-n) \rightarrow I^{*} \rightarrow 0$.

Thus $I$ does not have a linear resolution, but $I$ and $R=A / I$ are of homogeneous type. By the same argument as in the preceding example, one can prove that depth $S(m)=n^{2}+2 n-2$.

\section{REFERENCES}

1. M. F. Atiyah and J. Mac Donald, Introduction to commutative algebra, Addison-Wesley, 1969.

2. M. Brundu, Piattezza normale e isomolteplicita, Rend. Sem. Mat. Univ. Politec. Torino 40 (1982).

3. D. Eisenbud and S. Goto, Linear free resolutions and minimal multiplicity, J. Algebra 88 (1984), $89-133$.

4. R. Gebauer and H. Kredel, Buchberger's algorithm for constructing canonical bases (Gröhner bases) for polynomial ideals, Program Documentation, Univ. Heidelberg, 1983.

5. J. Herzog and M. Kühl, On the Bettinumbers of finite pure and linear resolutions, Comm. Algebra 12 (13), (1984), 1627-1646.

6. J. Herzog, A. Simis and W. Vasconcelos, Koszul homology and blowing-up rings, Commutative Algebra (Proc. Trento Conf.), Dekker, New York, 1983, pp. 79-169.

7. C. Huneke and M. E. Rossi, The dimension and components of symmetric algebras, J. Algebra (to appear).

8. L. Robbiano, Coni tangenti a singolarita razionali, Curve Algebriche, Istituto di Analisi Globale. Firenze, 1981. 
9. L. Robbiano and G. Valla, Free resolutions for special tangent cones, Commutative Algebra (Proc. Trento Conf.), Dekker, New York, 1983.

10. On the equations defining tangent cones, Math. Proc. Cambridge Philos. Soc. 88 (1980), $281-297$.

11. M. E. Rossi, Sulle algebre di Rees e simmetrica di un ideale, Le Matematiche 34 (1979), 1-2.

12. J. Sally, On the associated graded ring of a local Cohen-Macaulay ring, J. Math. Kyoto Univ. 17 (1977), 19-21.

13. P. Schenzel, Über die freien Auflösungen extremaler Cohen-Macaulay-Ringe, J. Algebra 64 (1980), 93-101.

14. P. Valabrega and G. Valla, Form rings and regular sequences, Nagoya Math. J. 72 (1978), 93-101.

Fachbereich Mathematik, Universität ESSEN, D - 4300, EsSEn 1, West Germany (Current address of J. Herzog)

Istituto di Matematica, 16132 Genova, Italy (Current address of M. Rossi and G. Valla) 